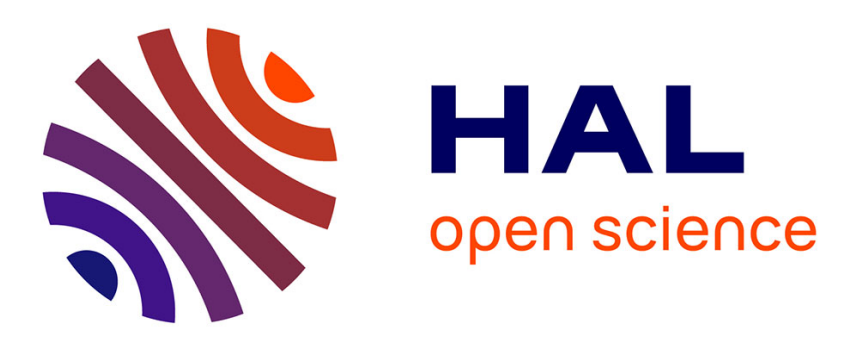

\title{
Effect of interpolation on noise propagation from images to DIC displacement maps
}

\author{
Benoît Blaysat, Michel Grediac, Frédéric Sur
}

\section{To cite this version:}

Benoît Blaysat, Michel Grediac, Frédéric Sur. Effect of interpolation on noise propagation from images to DIC displacement maps. International Journal for Numerical Methods in Engineering, 2016, 108 (3), pp.213-232. 10.1002/nme.5212 . hal-01255944

\section{HAL Id: hal-01255944 \\ https://inria.hal.science/hal-01255944}

Submitted on 12 Feb 2016

HAL is a multi-disciplinary open access archive for the deposit and dissemination of scientific research documents, whether they are published or not. The documents may come from teaching and research institutions in France or abroad, or from public or private research centers.
L'archive ouverte pluridisciplinaire $\mathbf{H A L}$, est destinée au dépôt et à la diffusion de documents scientifiques de niveau recherche, publiés ou non, émanant des établissements d'enseignement et de recherche français ou étrangers, des laboratoires publics ou privés. 


\title{
Effect of interpolation on noise propagation from images to DIC displacement maps
}

\author{
Benoît Blaysat, Michel GrédiaC ${ }^{\dagger}$ Frédéric SuR ${ }^{\ddagger}$
}

\begin{abstract}
This paper introduces and validates a new prediction of sensor noise propagation from images of randomly marked surfaces to displacement maps obtained by Digital Image Correlation (DIC). Images are indeed often affected by sensor noise, which propagates to DIC output. We consider here the 2D global DIC (G-DIC), for which this output is the inplane displacement calculated at a set of nodes. Predictive formula for the resolution of the displacement at these nodes is already available in the literature. The contribution of the present paper is to revisit this formula to take into account the interpolation required by sub-pixel displacement. A generalization is also proposed to predict the displacement resolution throughout the field of view. It is then extended to several kinds of DIC. The correlation procedure is thoroughly described in order to emphasize the role of the interpolation. A numerical assessment on synthetic data validates the new prediction and shows the improvement brought about by the proposed formula.
\end{abstract}

Keywords: Digital image correlation, displacement maps, measurement resolution, resolution prediction

This is the author-manuscript version of:

B. Blaysat, M. Grédiac, F. Sur. Effect of interpolation on noise propagation from images to DIC displacement maps. International Journal for Numerical Methods in Engineering, Wiley, 2016.

DOI: $10.1002 / \mathrm{nme} .5212$

\footnotetext{
${ }^{*}$ Corresponding author. Clermont Université, Université Blaise Pascal, Institut Pascal, UMR CNRS 6602, BP 10448, 63000 Clermont-Ferrand, France

${ }^{\dagger}$ Clermont Université, Université Blaise Pascal, Institut Pascal, UMR CNRS 6602, BP 10448, 63000 ClermontFerrand, France

${ }^{\ddagger}$ Laboratoire Lorrain de Recherche en Informatique et ses Applications, UMR CNRS 7503, Université de Lorraine, CNRS, INRIA projet Magrit, Campus Scientifique, BP 239, 54506 Vandœuvre-lès-Nancy Cedex, France
} 


\section{Introduction}

In experimental mechanics, Digital Image Correlation (DIC) is a method that allows to measure displacement maps of material surfaces using images acquired during specimen testing. Since the seminal work of Sutton [26, 6], DIC has spread out in the experimental mechanics community thanks to the increase of computing power and the decrease of its cost. Although the method is now thirty years old, improvements are regularly proposed, either following camera or computer technologies advances $([29,1]$ for instance), or by introducing new numerical approaches $([3,19$, $5,17,7,34]$ for instance).

Assessing the metrological performance of DIC is a major issue, this technique being expected to be a reliable measuring tool. DIC is indeed based on images of the specimen surface, and these images are affected by various phenomena, among which sensor noise. This noise propagates from images to DIC outputs, which are therefore also affected. The resolution, defined in [13] as the "smallest change in a quantity being measured that causes a perceptible change in the corresponding indication", and interpreted as "the smallest quantity that emerges from the noise floor" in [9], should be estimated and provided with the measurement itself. This paper focuses on the displacement resolution of DIC outputs, which is defined here as the standard deviation of the noise that corrupts the displacement, as in [9].

Since the first study by Sutton [25], which tackled the resolution issue, various predictive formulas for the displacement resolution have been provided in the literature $[22,2,3,20,18$, $19,12,8,32,14,31]$.

The larger part of these references is related to the local versions of DIC $[2,32,21,30,14,31$, $28]$. Only $[30,14,31,28]$ propose predictions that take the gray level interpolation due to the sub-pixel displacement into account. Nevertheless, the prediction in [30] is only valid for a pure subset translation; the prediction of $[14,31]$ deals with stereo-DIC effects. It is not validated with non-constant displacement field. The formula proposed in [28] mainly focuses on the bias.

The other part of these references is related to the global versions of DIC $[3,20,18,19,12,8]$. References $[3,20]$ propose an estimate of the average displacement resolution in the case of Finite Element-based Global DIC. This principle has been extended to nodal displacement resolution in $[18,19,12]$, or to other post-processed quantities [8]. However, none of these references proposes a detailed study which thoroughly validates these introduced predictions. Moreover, the effect of the sub-pixel displacement is not taken into account in any of these predictions.

In this context, we propose here to revisit the prediction of the measurement resolution of [18] to account for this interpolation. In addition, this predicted resolution corresponds to the degrees of freedom (DoF) resolution. These DoF correspond in turn to the displacement resolution in the particular case of Finite Element based Global-DIC approaches. This paper aims at introducing a generalized displacement resolution prediction that (i) accounts for sub-pixel displacement and (ii) offers pixelwise displacement resolution maps. The introduced formula generalizes a formula available in the literature [18], and the improvement brought about is highlighted. It is worth mentioning that realistic image acquisitions do not satisfy the assumptions usually required for deriving uncertainty formulas. For example, sensor noise is signal-dependent and cannot be considered as a Gaussian white noise (for more details, see [23] or [4]). Consequently, we focus here with the numerical assessment of the displacement resolution deduced from synthetic images, in which noise is perfectly known a priori and characterized. Before concluding, we give the main guidelines for a proper application of the proposed prediction. 


\section{Methods}

This section aims at introducing a generalized formula for predicting the displacement resolution. First, a short digest of the DIC method is recalled. It gives a thorough description of the formalism needed to elaborate the prediction of the DoF standard deviation. Noisy data and sub-pixel displacements are taken into account. This prediction is also compared with that proposed in [18]. Finally, a generalized formula is proposed. It leads to displacement resolution maps which are defined pixelwise. We discuss here resolution prediction within the framework of G-DIC. The local version of DIC can also be formulated as a particular case of G-DIC. Consequently, our prediction can also be considered in this case, see [4]. The reader interested in comparing local and global DIC is invited to refer to [12, 16, 33] for instance. This comparison is not within the scope of the present paper.

\subsection{DIC basics}

DIC aims at estimating the real, unknown, displacement field that occurs between two images of the same material surface, namely the reference image $(f)$ and the current image $(g)$. $(g)$ is acquired after loading the specimen. Let $\underline{U}$ be the sought real displacement and $\underline{u}$ be its estimate. The backbone of the method is the conservation of the intensity over a Region of Interest (RoI) while testing. In other words, the gray level values are assumed to be translated from their initial location in the reference image $(f)$ to their updated location in the current one $(g)$ :

$$
\forall \underline{x} \in \operatorname{RoI}, \quad f(\underline{x}) \approx g(\underline{x}+\underline{U}(\underline{x}))
$$

$\underline{U}$ is here the displacement of the material surface. It is observed from the sensor point of view. $\underline{x} \in \mathrm{RoI}$ is expressed in the sensor coordinate system denoted $\left(\underline{O} ; \underline{e}_{1}, \underline{e}_{2}\right)$. With DIC, an approximated kinematics space $\mathcal{U}$ is introduced. The sum over the RoI pixels of the squared residual of Eq.(1) is then minimized in order to determine the optimized displacement $\underline{u}^{\text {opti }}$ :

$$
\underline{u}^{\mathrm{opti}}=\underset{\underline{u} \in \mathcal{U}}{\operatorname{Argmin}}\left\{\sum_{\underline{x}_{\mathrm{p}} \in \operatorname{RoI}}\left(f\left(\underline{x}_{\mathrm{p}}\right)-g\left(\underline{x}_{\mathrm{p}}+\underline{u}\left(\underline{x}_{\mathrm{p}}\right)\right)\right)^{2}\right\}
$$

In practice, the kinematics space $\mathcal{U}$ is generally defined as a vector space of dimension $N$, built up from a basis of functions denoted $\left(\underline{\varphi}_{i}\right)_{1 \leq i \leq N}$. Any approximated displacement $\underline{u}$ is thus defined as a combination of the $N$ functions $\left(\underline{\varphi}_{i}\right)_{1 \leq i \leq N}$. Introducing the DoF vector $\boldsymbol{\lambda}=\left(\lambda_{i}\right)_{1 \leq i \leq N}$ leads to:

$$
\forall \underline{x} \in \operatorname{RoI}, \quad \underline{u}(\underline{x}) \equiv \underline{u}(\underline{x}, \boldsymbol{\lambda})=\sum_{i=1}^{N} \lambda_{i} \underline{\varphi}_{i}(\underline{x}) .
$$

The problem can thus be reformulated with the DoF vector. Solving Eq.(2) is equivalent to solve

$$
\boldsymbol{\lambda}^{\mathrm{opti}}=\underset{\boldsymbol{\lambda}}{\operatorname{Argmin}}\left\{\sum_{\underline{x}_{\mathrm{p}} \in \mathrm{RoI}}\left(r\left(\underline{x}_{\mathrm{p}}, \boldsymbol{\lambda}\right)\right)^{2}\right\},
$$

where the residual writes

$$
r\left(\underline{x}_{\mathrm{p}}, \boldsymbol{\lambda}\right)=f\left(\underline{x}_{\mathrm{p}}\right)-g\left(\underline{x}_{\mathrm{p}}+\sum_{i=1}^{N} \lambda_{i} \underline{\varphi}_{i}\left(\underline{x}_{\mathrm{p}}\right)\right) .
$$


Let the vector $\boldsymbol{r}(\boldsymbol{\lambda})$ be such that its $i$-th component is such that $[\boldsymbol{r}(\boldsymbol{\lambda})]_{i}=r\left(\underline{x}_{\mathrm{i}}, \boldsymbol{\lambda}\right)$, where $\underline{x}_{\mathrm{i}}$ spans the RoI. Eq.(4) becomes:

$$
\boldsymbol{\lambda}^{\mathrm{opti}}=\underset{\boldsymbol{\lambda}}{\operatorname{Argmin}}\left\{\|\boldsymbol{r}(\boldsymbol{\lambda})\|^{2}\right\} .
$$

where $\|$.$\| is the Euclidean norm of any vector.$

This minimization problem is solved using a Gauss-Newton scheme. It consists, from an initial guess $\boldsymbol{\lambda}^{0}$, in iteratively minimizing $\left\|\boldsymbol{r}\left(\boldsymbol{\lambda}^{\mathrm{it}+1}\right)\right\|^{2}$ with $\boldsymbol{\lambda}^{\mathrm{it}+1}=\boldsymbol{\lambda}^{\text {it }}+\boldsymbol{\Delta} \boldsymbol{\lambda}^{\text {it }}$, where $\boldsymbol{\Delta} \boldsymbol{\lambda}^{\text {it }}$ is assumed to be small with respect to $\lambda^{\text {it }}$. Under these conditions, $\Delta \lambda^{\text {it }}$ can be computed by approximating $\boldsymbol{r}\left(\boldsymbol{\lambda}^{\text {it }}+\boldsymbol{\Delta} \boldsymbol{\lambda}^{\text {it }}\right)$ by its first-order Taylor expansion:

$$
\boldsymbol{r}\left(\boldsymbol{\lambda}^{\mathrm{it}}+\boldsymbol{\Delta} \boldsymbol{\lambda}^{\mathrm{it}}\right)=\boldsymbol{r}\left(\boldsymbol{\lambda}^{\mathrm{it}}\right)+\boldsymbol{J}_{\boldsymbol{r}}\left(\boldsymbol{\lambda}^{\mathrm{it}}\right) \boldsymbol{\Delta} \boldsymbol{\lambda}^{\mathrm{it}},
$$

where $\boldsymbol{J}_{\boldsymbol{r}}\left(\boldsymbol{\lambda}^{\mathrm{it}}\right)$ is the Jacobian matrix of $\boldsymbol{r}$ at $\boldsymbol{\lambda}^{\text {it }}$. It is calculated with the chain rule as follows

$$
\begin{aligned}
{\left[\boldsymbol{J}_{\boldsymbol{r}}\left(\boldsymbol{\lambda}^{\mathrm{it}}\right)\right]_{i j} } & =\frac{\partial}{\partial[\boldsymbol{\lambda}]_{j}}\left[\boldsymbol{r}\left(\boldsymbol{\lambda}^{\mathrm{it}}\right)\right]_{i}=\frac{\partial r}{\partial \lambda_{j}}\left(\underline{x}_{\mathrm{i}}, \boldsymbol{\lambda}^{\mathrm{it}}\right) \\
& =-\underline{\nabla}(g)\left(\underline{x}_{\mathrm{i}}+\underline{u}\left(\underline{x}_{\mathrm{i}}, \boldsymbol{\lambda}^{\mathrm{it}}\right)\right) \cdot \frac{\partial \underline{u}}{\partial \lambda_{j}}\left(\underline{x}_{\mathrm{i}}, \boldsymbol{\lambda}^{\mathrm{it}}\right) \\
& =-\underline{\nabla}(g)\left(\underline{x}_{\mathrm{i}}+\underline{u}\left(\underline{x}_{\mathrm{i}}, \boldsymbol{\lambda}^{\mathrm{it}}\right)\right) \cdot \underline{\varphi}_{j}\left(\underline{x}_{\mathrm{i}}\right)
\end{aligned}
$$

As detailed in $[27,15]$, it is commonly accepted to substitute the gradient of the current image $(g)$ evaluated at the updated coordinates $\underline{x}_{\mathrm{i}}+\underline{u}\left(\underline{x}_{\mathrm{i}}\right)$ by the gradient of the reference image $(f)$ when small displacement occurs. This leads to the following simplification

$$
\underline{\nabla}(g)\left(\underline{x}_{\mathrm{i}}+\underline{u}\left(\underline{x}_{\mathrm{i}}, \lambda^{\mathrm{it}}\right)\right) \approx \underline{\nabla}(f)\left(\underline{x}_{\mathrm{i}}\right)
$$

which gives

$$
\boldsymbol{J}_{r}\left(\boldsymbol{\lambda}^{\mathrm{it}}\right) \approx-\boldsymbol{L}, \quad \text { with } \quad[\boldsymbol{L}]_{i j}=\underline{\nabla}(f)\left(\underline{x}_{\mathrm{i}}\right) \underline{\varphi}_{j}\left(\underline{x}_{\mathrm{i}}\right) .
$$

As a consequence, the residual is approximated here by $\boldsymbol{r}\left(\boldsymbol{\lambda}^{\mathrm{it}}+\boldsymbol{\Delta} \lambda^{\mathrm{it}}\right)=\boldsymbol{r}\left(\boldsymbol{\lambda}^{\mathrm{it}}\right)-\boldsymbol{L} \boldsymbol{\Delta} \lambda^{\mathrm{it}}$. The minimization of $\left\|\boldsymbol{r}\left(\boldsymbol{\lambda}^{\mathrm{it}}\right)-\boldsymbol{L} \boldsymbol{\Delta} \lambda^{\mathrm{it}}\right\|^{2}$ is an ordinary least squares problem, which gives $\boldsymbol{\Delta} \boldsymbol{\lambda}^{\mathrm{it}}$ as the solution of the normal equation:

$$
\boldsymbol{L}^{T} \boldsymbol{L} \boldsymbol{\Delta} \boldsymbol{\lambda}^{\mathrm{it}}=\boldsymbol{L}^{T} \boldsymbol{r}\left(\boldsymbol{\lambda}^{\mathrm{it}}\right)
$$

The increment $\Delta \boldsymbol{\lambda}^{\mathrm{it}}$ is thus computed as follows

$$
\boldsymbol{\Delta} \boldsymbol{\lambda}^{\mathrm{it}}=\boldsymbol{M}^{-1} \boldsymbol{b}^{\mathrm{it}}, \quad \text { with } \quad\left\{\begin{array}{l}
\boldsymbol{M}=\boldsymbol{L}^{T} \boldsymbol{L} \\
\boldsymbol{b}^{\mathrm{it}}=\boldsymbol{L}^{T} \boldsymbol{r}\left(\boldsymbol{\lambda}^{\mathrm{it}}\right)
\end{array}\right.
$$

In this modified Gauss-Newton algorithm, the DoF vector is incremented by $\boldsymbol{\Delta} \boldsymbol{\lambda}^{\text {it }}$ until $\left\|\boldsymbol{b}^{\text {it }}\right\|$ reaches some threshold value. This value is chosen here to be equal to $10^{-5} \times\left\|\boldsymbol{b}^{0}\right\|$, where $\left\|\boldsymbol{b}^{0}\right\|$ is defined as the initial value of $\left\|\boldsymbol{b}^{\mathrm{it}}\right\|$. This gives good results in the considered cases. Thanks to Eq.(11) which simplifies the calculation of the Jacobian, the tangent operator $\boldsymbol{M}$ is constant for all iterations. It can thus be computed beforehand, which dramatically reduces the computational cost of the DIC solving. What follows focuses on the determination of $\boldsymbol{b}^{\text {it }}$. For this purpose, the column matrix notation is also introduced for images $(f)$ and $(g): \boldsymbol{f}$ and $\boldsymbol{g}$ are respectively the vectors of their pixel gray level values (for any $\underline{x}_{\mathrm{i}} \in \mathrm{RoI},[\boldsymbol{f}]_{i}=f\left(\underline{x}_{\mathrm{i}}\right)$ and $\left.[\boldsymbol{g}]_{i}=g\left(\underline{x}_{\mathrm{i}}\right)\right)$. The back-deformed image $\left(g_{\mathrm{BD}}(\boldsymbol{\lambda})\right)$ is also defined. It is the interpolated value of the current image $(g)$ at $\underline{x}_{\mathrm{p}}+\underline{u}\left(\underline{x}_{\mathrm{p}}, \boldsymbol{\lambda}\right)\left(i . e ., g_{\mathrm{BD}}\left(\underline{x}_{\mathrm{i}}, \boldsymbol{\lambda}\right)=g\left(\underline{x}_{\mathrm{i}}+\underline{u}\left(\underline{x}_{\mathrm{i}}, \boldsymbol{\lambda}\right)\right)\right)$. Vector $\boldsymbol{g}_{\mathrm{BD}}(\boldsymbol{\lambda})$ denotes $\left[\boldsymbol{g}_{\mathrm{BD}}(\boldsymbol{\lambda})\right]_{i}=g_{\mathrm{BD}}\left(\underline{x}_{\mathrm{i}}, \boldsymbol{\lambda}\right)$. With this notation, $\boldsymbol{b}^{\text {it }}$ writes

$$
\boldsymbol{b}^{\mathrm{it}}=\boldsymbol{L}^{T} \boldsymbol{r}\left(\boldsymbol{\lambda}^{\mathrm{it}}\right)=\boldsymbol{L}^{T}\left(\boldsymbol{f}-\boldsymbol{g}_{\mathrm{BD}}\left(\boldsymbol{\lambda}^{\mathrm{it}}\right)\right)
$$


Since $(g)$ is only known at the pixel locations, the calculation of $\boldsymbol{g}_{\mathrm{BD}}(\boldsymbol{\lambda})$ requires an interpolation. Two cases are considered here: bilinear and bicubic interpolations, but only the first one is detailed. The bicubic case is discussed in Section 3 which is devoted to the validation of the prediction. The interpolation is formulated here by splitting at each pixel $\underline{x}_{\mathrm{i}}$ the displacement $\underline{u}\left(\underline{x}_{\mathrm{i}}, \boldsymbol{\lambda}\right)$ into its sub-pixel part $\alpha_{\mathrm{i}}(\boldsymbol{\lambda}) \underline{e}_{1}+\beta_{\mathrm{i}}(\boldsymbol{\lambda}) \underline{e}_{2}$, and its pixel part $\mathrm{U}_{\mathrm{i}}(\boldsymbol{\lambda}) \underline{e}_{1}+\mathrm{v}_{\mathrm{i}}(\boldsymbol{\lambda}) \underline{e}_{2}$ such that $\underline{u}\left(\underline{x}_{\mathrm{i}}, \boldsymbol{\lambda}\right)=\left(\mathrm{U}_{\mathrm{i}}(\boldsymbol{\lambda})+\alpha_{\mathrm{i}}(\boldsymbol{\lambda})\right) \underline{e}_{1}+\left(\mathrm{V}_{\mathrm{i}}(\boldsymbol{\lambda})+\beta_{\mathrm{i}}(\boldsymbol{\lambda})\right) \underline{e}_{2}$. With this notation, for all $\boldsymbol{\lambda}$, one has $\left(\alpha_{\mathrm{i}}(\boldsymbol{\lambda}), \beta_{\mathrm{i}}(\boldsymbol{\lambda})\right) \in[0,1]^{2}$ and $\left(\mathrm{U}_{\mathrm{i}}(\boldsymbol{\lambda}), \mathrm{v}_{\mathrm{i}}(\boldsymbol{\lambda})\right) \in \mathbb{Z}^{2}$.

For any $\underline{x}_{\mathrm{i}}$ in the RoI, the bilinearly interpolated value of $\left[\boldsymbol{g}_{\mathrm{BD}}\right]_{i}$ can be expressed as follows

$$
\begin{aligned}
{\left[\boldsymbol{g}_{\mathrm{BD}}(\boldsymbol{\lambda})\right]_{i}=\left(1-\alpha_{\mathrm{i}}(\boldsymbol{\lambda})\right)\left(1-\beta_{\mathrm{i}}(\boldsymbol{\lambda})\right) g\left(\underline{x}_{\mathrm{i}}+\underline{\mathrm{U}}_{\mathrm{i}}(\boldsymbol{\lambda})\right)+\alpha_{\mathrm{i}}(\boldsymbol{\lambda})\left(1-\beta_{\mathrm{i}}(\boldsymbol{\lambda})\right) g\left(\underline{x}_{\mathrm{i}}+\underline{\mathrm{U}}_{\mathrm{i}}(\boldsymbol{\lambda})+\underline{e}_{1}\right) } \\
+\left(1-\alpha_{\mathrm{i}}(\boldsymbol{\lambda})\right) \beta_{\mathrm{i}}(\boldsymbol{\lambda}) g\left(\underline{x}_{\mathrm{i}}+\underline{\mathrm{U}}_{\mathrm{i}}(\boldsymbol{\lambda})+\underline{e}_{2}\right)+\alpha_{\mathrm{i}}(\boldsymbol{\lambda}) \beta_{\mathrm{i}}(\boldsymbol{\lambda}) g\left(\underline{x}_{\mathrm{i}}+\underline{\mathrm{U}}_{\mathrm{i}}(\boldsymbol{\lambda})+\underline{e}_{1}+\underline{e}_{2}\right) .
\end{aligned}
$$

Let I, II, III and IV be the indices such that

$$
\left\{\begin{array}{l}
{[\boldsymbol{g}]_{1}=g\left(\underline{x}_{\mathrm{i}}+\underline{\mathrm{U}}_{\mathrm{i}}(\boldsymbol{\lambda})\right)} \\
{[\boldsymbol{g}]_{I \mathrm{I}}=g\left(\underline{x}_{\mathrm{i}}+\underline{\mathrm{U}}_{\mathrm{i}}(\boldsymbol{\lambda})+\underline{e}_{1}\right)} \\
\left.[\boldsymbol{g}]_{\mathrm{III}}=g \underline{x}_{\mathrm{i}}+\underline{\mathrm{u}}_{\mathrm{i}}(\boldsymbol{\lambda})+\underline{e}_{2}\right) \\
{[\boldsymbol{g}]_{\mathrm{IV}}=g\left(\underline{x}_{\mathrm{i}}+\underline{\mathrm{u}}_{\mathrm{i}}(\boldsymbol{\lambda})+\underline{e}_{1}+\underline{e}_{2}\right)}
\end{array} .\right.
$$

With this notation, Eq.(16) leads to

$$
\begin{array}{r}
{\left[\boldsymbol{g}_{\mathrm{BD}}(\boldsymbol{\lambda})\right]_{i}=\left(1-\alpha_{\mathrm{i}}(\boldsymbol{\lambda})\right)\left(1-\beta_{\mathrm{i}}(\boldsymbol{\lambda})\right)[\boldsymbol{g}]_{\mathrm{I}}+\alpha_{\mathrm{i}}(\boldsymbol{\lambda})\left(1-\beta_{\mathrm{i}}(\boldsymbol{\lambda})\right)[\boldsymbol{g}]_{\mathrm{II}}} \\
+\left(1-\alpha_{\mathrm{i}}(\boldsymbol{\lambda})\right) \beta_{\mathrm{i}}(\boldsymbol{\lambda})[\boldsymbol{g}]_{\mathrm{III}}+\alpha_{\mathrm{i}}(\boldsymbol{\lambda}) \beta_{\mathrm{i}}(\boldsymbol{\lambda})[\boldsymbol{g}]_{\mathrm{IV}} .
\end{array}
$$

Let $\boldsymbol{P}(\boldsymbol{\lambda})$ be the interpolation matrix, such that:

$$
\boldsymbol{g}_{\mathrm{BD}}(\boldsymbol{\lambda})=\boldsymbol{P}(\boldsymbol{\lambda}) \boldsymbol{g}
$$

$$
\text { with } \quad \forall \mathrm{i} \in\left\{1, \ldots, N_{p}\right\},\left\{\begin{array}{l}
\forall \mathrm{j} \in\left\{1, \ldots, N_{p}\right\} \backslash\{\mathrm{I}, \mathrm{II}, \mathrm{III}, \mathrm{IV}\},[\boldsymbol{P}(\boldsymbol{\lambda})]_{i j}=0 \\
{[\boldsymbol{P}(\boldsymbol{\lambda})]_{i \mathrm{I}}=\left(1-\alpha_{\mathrm{i}}(\boldsymbol{\lambda})\right)\left(1-\beta_{\mathrm{i}}(\boldsymbol{\lambda})\right)} \\
{[\boldsymbol{P}(\boldsymbol{\lambda})]_{i l l}=\alpha_{\mathrm{i}}(\boldsymbol{\lambda})\left(1-\beta_{\mathrm{i}}(\boldsymbol{\lambda})\right)} \\
{[\boldsymbol{P}(\boldsymbol{\lambda})]_{i l l I}=\left(1-\alpha_{\mathrm{i}}(\boldsymbol{\lambda})\right) \beta_{\mathrm{i}}(\boldsymbol{\lambda})} \\
{[\boldsymbol{P}(\boldsymbol{\lambda})]_{i \mathrm{IV}}=\alpha_{\mathrm{i}}(\boldsymbol{\lambda}) \beta_{\mathrm{i}}(\boldsymbol{\lambda})}
\end{array}\right.
$$

Using this notation, $\boldsymbol{b}^{\text {it }}$ reads as follows

$$
\boldsymbol{b}^{\mathrm{it}}=\boldsymbol{L}^{T} \boldsymbol{r}\left(\boldsymbol{\lambda}^{\mathrm{it}}\right)=\boldsymbol{L}^{T}\left(\boldsymbol{f}-\boldsymbol{P}\left(\boldsymbol{\lambda}^{\mathrm{it}}\right) \boldsymbol{g}\right)
$$

\subsection{Consequence of sensor noise}

The derivation proposed above is valid for noise-free images. It turns out that real images are impaired by sensor noise. DIC outputs are thus also affected. In order to highlight how noise propagates from images to displacement maps, noisy reference $(\tilde{f})$ and current $(\tilde{g})$ images are introduced:

$$
\forall \underline{x}_{\mathrm{p}} \in \operatorname{RoI}\left\{\begin{array}{l}
\tilde{f}\left(\underline{x}_{\mathrm{p}}\right)=f\left(\underline{x}_{\mathrm{p}}\right)+n_{f}\left(\underline{x}_{\mathrm{p}}\right) \\
\tilde{g}\left(\underline{x}_{\mathrm{p}}\right)=g\left(\underline{x}_{\mathrm{p}}\right)+n_{g}\left(\underline{x}_{\mathrm{p}}\right)
\end{array}\right.
$$

where $(f)$ and $(g)$ are unknown noise-free images, and noises $\left(n_{f}\right)$ and $\left(n_{g}\right)$ are defined under the following hypothesis:

Hypothesis 1 Sensor noises $\left(n_{f}\right)$ and $\left(n_{g}\right)$ are assumed to be 0 -mean Gaussian white noises of standard deviation $\sigma_{f}$. 
It is important to note that, as for any white noise, $\sigma_{f}$ is constant over the RoI. Let $\boldsymbol{n}_{f}$ (resp. $\boldsymbol{n}_{g}$ ) be the vector made of pixel gray level values of $\left(n_{f}\right)$ (resp. $\left(n_{g}\right)$ ). Thanks to Hyp.(1), $\boldsymbol{n}_{f}$ (resp. $\boldsymbol{n}_{g}$ ) is a Gaussian random vector. Consequently, the following properties are satisfied:

$$
\left\{\begin{array}{l}
\text { mean : }\left\langle\boldsymbol{n}_{f}\right\rangle=\left\langle\boldsymbol{n}_{g}\right\rangle=\mathbf{0}, \\
\text { covariance matrix }: \boldsymbol{\Sigma}_{n_{f}}=\boldsymbol{\Sigma}_{n_{g}}=\left(\sigma_{f}\right)^{2} \times \boldsymbol{I} \quad(\boldsymbol{I} \text { being the identity matrix }) .
\end{array}\right.
$$

Remark 2.1 It is important to point out that camera sensor noise is actually not a white noise as assumed in Hyp.(1). This hypothesis can consequently be relaxed by assuming sensor noises $\left(n_{f}\right)$ and $\left(n_{g}\right)$ to be $0-$ mean Gaussian noises of standard deviation $\sigma_{f}\left(\underline{x}_{\mathrm{p}}\right)$, which varies pixelwise. The covariance matrices $\boldsymbol{\Sigma}_{n_{f}}$ and $\boldsymbol{\Sigma}_{n_{g}}$ of the Gaussian random vectors $\boldsymbol{n}_{f}$ and $\boldsymbol{n}_{g}$ would consequently be diagonal such that $\forall i,\left[\boldsymbol{\Sigma}_{n_{f}}\right]_{i i}=\left[\boldsymbol{\Sigma}_{n_{g}}\right]_{i i}=\sigma_{f}\left(\underline{x}_{i}\right)^{2}$. For the sake of simplicity, the simple case of white noises (i.e., Hyp.(1)) is considered in what follows.

With noisy images, the optimal DoF writes :

$$
\tilde{\boldsymbol{\lambda}}^{\text {opti }}=\underset{\boldsymbol{\lambda}}{\operatorname{Argmin}}\left\{\|\tilde{\boldsymbol{r}}(\boldsymbol{\lambda})\|^{2}\right\} .
$$

where subscript " " denotes quantities subsequently affected by noise. With these notations, the residual becomes:

$$
\tilde{\boldsymbol{r}}(\tilde{\boldsymbol{\lambda}})=\tilde{\boldsymbol{f}}-\tilde{\boldsymbol{g}}_{\mathrm{BD}}(\tilde{\boldsymbol{\lambda}})=\boldsymbol{f}+\boldsymbol{n}_{f}-\boldsymbol{P}(\tilde{\boldsymbol{\lambda}})\left(\boldsymbol{g}+\boldsymbol{n}_{g}\right)
$$

Noise propagates through the modified Gauss-Newton algorithm, which minimizes now the residual of the noisy images.

Let $\delta \boldsymbol{\lambda}$ be the perturbation of the optimal values of the DoF vector $\tilde{\boldsymbol{\lambda}}^{\text {opti }}$ caused by acquisition noise, i.e., $\tilde{\boldsymbol{\lambda}}^{\text {opti }}=\boldsymbol{\lambda}^{\text {opti }}+\boldsymbol{\delta} \boldsymbol{\lambda}$. In what follows, $\boldsymbol{\lambda}^{\text {opti }}$ is simply denoted $\boldsymbol{\lambda}$. Once the convergence criterion is reached, the following hypothesis is assumed:

Hypothesis $2 \delta \boldsymbol{\lambda}$ is assumed to be small enough with respect to the optimized DoF values $\boldsymbol{\lambda}$ themselves to allow the first-order Taylor approximation:

$$
\tilde{\boldsymbol{r}}\left(\tilde{\boldsymbol{\lambda}}^{\mathrm{opti}}\right)=\tilde{\boldsymbol{r}}(\boldsymbol{\lambda})+\boldsymbol{J}_{\tilde{\boldsymbol{r}}}(\boldsymbol{\lambda}) \boldsymbol{\delta} \boldsymbol{\lambda}
$$

$\boldsymbol{J}_{\tilde{\boldsymbol{r}}}(\boldsymbol{\lambda})$ is the Jacobian matrix of the residual $\tilde{\boldsymbol{r}}$ affected by noise at $\boldsymbol{\lambda}$. From Eq.(10), it satisfies at any pixel $\underline{x}_{\mathrm{i}}$ and for any $j$ in $\{1, \ldots, N\}$,

$$
\left[\boldsymbol{J}_{\tilde{\boldsymbol{r}}}(\boldsymbol{\lambda})\right]_{i j}=-\underline{\nabla}(\tilde{g})\left(\underline{x}_{\mathrm{i}}+\underline{u}\left(\underline{x}_{\mathrm{i}}, \boldsymbol{\lambda}\right)\right) \cdot \underline{\varphi}_{j}\left(\underline{x}_{\mathrm{i}}\right) .
$$

At convergence, we have

$$
\left\|\tilde{\boldsymbol{r}}\left(\tilde{\boldsymbol{\lambda}}^{\mathrm{opti}}\right)\right\|^{2} \approx 0
$$

The perturbation $\boldsymbol{\delta} \boldsymbol{\lambda}$ satisfies therefore:

$$
\boldsymbol{J}_{\tilde{\boldsymbol{r}}}(\boldsymbol{\lambda})^{T} \boldsymbol{J}_{\tilde{\boldsymbol{r}}}(\boldsymbol{\lambda}) \boldsymbol{\delta} \boldsymbol{\lambda}+\boldsymbol{J}_{\tilde{\boldsymbol{r}}}(\boldsymbol{\lambda})^{T} \tilde{\boldsymbol{r}}(\boldsymbol{\lambda})=0 .
$$

Introducing the same matrix notation as in the noiseless case, Eq.(28) writes:

$$
\tilde{\boldsymbol{M}}^{-1} \boldsymbol{\delta} \boldsymbol{\lambda}=\tilde{\boldsymbol{b}}, \quad \text { with } \quad\left\{\begin{array}{l}
\tilde{\boldsymbol{M}}=\boldsymbol{J}_{\tilde{\boldsymbol{r}}}(\boldsymbol{\lambda})^{T} \boldsymbol{J}_{\tilde{\boldsymbol{r}}}(\boldsymbol{\lambda}) \\
\tilde{\boldsymbol{b}}=-\boldsymbol{J}_{\tilde{\boldsymbol{r}}}(\boldsymbol{\lambda})^{T}(\tilde{\boldsymbol{f}}-\boldsymbol{P}(\boldsymbol{\lambda}) \tilde{\boldsymbol{g}})
\end{array}\right.
$$


A complete analysis of noise propagation would necessitate to take into account the uncertainty in the estimation of $\underline{\nabla}(\tilde{g})$. This uncertainty depends on the numerical scheme used to calculate the images gradient, and thus on the Jacobian $\boldsymbol{J}_{\tilde{\boldsymbol{r}}}(\boldsymbol{\lambda})$ and the matrix $\tilde{\boldsymbol{M}}$ in Eq.(29). Since $\tilde{M}^{-1}$ does not linearly depends on $\underline{\nabla}(\tilde{g})$, a standard possibility is to use first order approximations. Addressing this question would require a large body of calculations. It is consequently beyond the scope of the present paper. The interested reader can found further information for instance in [24], where a comparable analysis is led in another context. Matrix $\tilde{\boldsymbol{M}}$ is therefore elaborated as the matrix $\boldsymbol{M}$ in the following way:

$$
\tilde{\boldsymbol{M}}=\tilde{\boldsymbol{L}}^{T} \tilde{\boldsymbol{L}} \text { with }[\tilde{\boldsymbol{L}}]_{i j}=\underline{\nabla}(\tilde{f})\left(\underline{x}_{\mathrm{i}}\right) \underline{\varphi}_{j}\left(\underline{x}_{\mathrm{i}}\right)
$$

We will further simplify the calculation by introducing the following quantities:

Hypothesis 3 The noisy image gradients $\underline{\nabla} \tilde{f}$ and $\underline{\nabla} \tilde{g}$ are identified with their counterparts calculated for the noiseless images:

$$
\underline{\nabla} \tilde{f} \approx \underline{\nabla} f \quad \text { and } \quad \underline{\nabla} \tilde{g} \approx \underline{\nabla g}
$$

This assumption is carefully discussed in the section devoted to the numerical assessments.

As a consequence, $\tilde{\boldsymbol{M}} \approx \boldsymbol{M}$. Hence perturbation $\delta \boldsymbol{\lambda}$ defined in Eq.(25) satisfies:

$$
\boldsymbol{\delta} \boldsymbol{\lambda}=-\boldsymbol{M}^{-1}\left(\boldsymbol{J}_{\tilde{\boldsymbol{r}}}(\boldsymbol{\lambda})^{T}(\tilde{\boldsymbol{f}}-\boldsymbol{P}(\boldsymbol{\lambda}) \tilde{\boldsymbol{g}})\right) .
$$

Finally, the noise is supposed to have a small effect on the DIC solution itself. In other words, the hypothesis below holds:

Hypothesis 4 Once the convergence criterion is reached, we have $\boldsymbol{P}\left(\tilde{\lambda}^{\text {opti }}\right)=\boldsymbol{P}(\boldsymbol{\lambda})$ (resp. $\left.\boldsymbol{J}_{\tilde{\boldsymbol{r}}}\left(\tilde{\boldsymbol{\lambda}}^{\text {opti }}\right)=\boldsymbol{J}_{\tilde{\boldsymbol{r}}}(\boldsymbol{\lambda})\right)$. We introduce $\boldsymbol{P}=\boldsymbol{P}(\boldsymbol{\lambda})$ and $\boldsymbol{J}_{\tilde{\boldsymbol{r}}}=\boldsymbol{J}_{\tilde{\boldsymbol{r}}}(\boldsymbol{\lambda})$ to alleviate the notation. We obtain :

$$
\delta \boldsymbol{\lambda}=-\boldsymbol{M}^{-1} \cdot\left(\boldsymbol{J}_{\tilde{\boldsymbol{r}}}^{T} \cdot(\tilde{\boldsymbol{f}}-\boldsymbol{P} \cdot \tilde{\boldsymbol{g}})\right) .
$$

According to theses hypotheses, the DoF perturbation caused by sensor noise writes

$$
\begin{aligned}
\boldsymbol{\delta} \boldsymbol{\lambda} & =-\boldsymbol{M}^{-1}\left(\boldsymbol{J}_{\tilde{\boldsymbol{r}}}^{T}\left(\left(\boldsymbol{f}+\boldsymbol{n}_{f}\right)-\boldsymbol{P}\left(\boldsymbol{g}+\boldsymbol{n}_{g}\right)\right)\right) \\
& =-\boldsymbol{M}^{-1}\left(\boldsymbol{J}_{\tilde{\boldsymbol{r}}}^{T}(\boldsymbol{f}-\boldsymbol{P g})+\boldsymbol{J}_{\tilde{\boldsymbol{r}}}^{T}\left(\boldsymbol{n}_{f}-\boldsymbol{P} \boldsymbol{n}_{g}\right)\right) \\
& =\boldsymbol{A}(\boldsymbol{f}-\boldsymbol{P g})+\boldsymbol{A}\left(\boldsymbol{n}_{f}-\boldsymbol{P} \boldsymbol{n}_{g}\right) \text { with } \boldsymbol{A}=-\boldsymbol{M}^{-1} \boldsymbol{J}_{\tilde{\boldsymbol{r}}}^{T}
\end{aligned}
$$

The first term of Eq.(35), i.e., $\boldsymbol{A}(\boldsymbol{f}-\boldsymbol{P g})$, involves noiseless images $(f)$ and $(g)$ and corresponds to the measurement bias introduced by the interpolation scheme [21]. The property recalled below allows us to characterize the random DoF perturbation $\delta \lambda$ from the mean and covariance matrix of the random noise $\left(n_{f}\right)$ and $\left(n_{g}\right)[11]$ :

Proposition 2.1 If $\boldsymbol{x}$ is a Gaussian random vector of size $k$, mean $\langle\boldsymbol{x}\rangle$ and covariance matrix $\boldsymbol{\Sigma}_{\boldsymbol{x}}$, and if $\boldsymbol{C}$ is a matrix of size $l \times k$, then $\boldsymbol{y}=\boldsymbol{C . \boldsymbol { x }} . \boldsymbol{y}$ is a Gaussian random vector of size $l$, whose mean value $\langle\boldsymbol{x}\rangle$ and covariance matrix $\boldsymbol{\Sigma}_{\boldsymbol{y}}$ write $\langle\boldsymbol{y}\rangle=\boldsymbol{C} \cdot\langle\boldsymbol{x}\rangle$ and $\boldsymbol{\Sigma}_{\boldsymbol{y}}=\boldsymbol{C} \cdot \boldsymbol{\Sigma}_{\boldsymbol{x}} \cdot \boldsymbol{C}^{T}$, respectively. 
Moreover, $\left(n_{f}\right)$ and $\left(n_{g}\right)$ are two independent processes. Thus, using Prop.(2.1) and assuming Hyp.(3), the perturbation vector $\delta \boldsymbol{\lambda}$ satisfies the following statistical properties:

$$
\left\{\begin{array}{c}
\text { mean : }\langle\delta \boldsymbol{\lambda}\rangle=\boldsymbol{A}(\boldsymbol{f}+\boldsymbol{P} \boldsymbol{g}) \\
\text { covariance matrix : } \boldsymbol{\Sigma}_{\delta \boldsymbol{\lambda}}=\boldsymbol{A}\left(\sigma_{f}^{2} \times \boldsymbol{I}+\boldsymbol{P}\left(\sigma_{f}^{2} \times \boldsymbol{I}\right) \boldsymbol{P}^{T}\right) \boldsymbol{A}^{T} \\
=\sigma_{f}^{2} \times \boldsymbol{A}\left(\boldsymbol{I}+\boldsymbol{P} \boldsymbol{P}^{T}\right) \boldsymbol{A}^{T}
\end{array}\right.
$$

Eq.(36) also illustrates important properties of the back-deformed image. The properties of the back-deformed image noise is indeed embedded in it. They can be computed separately, based on Eq.(19) and using Prop.(2.1):

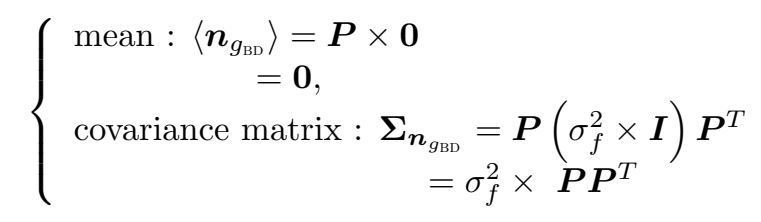

Finally, the standard deviation $\sigma_{\lambda_{i}}^{p}$ of the $i^{\text {th }}$ DoF can be calculated as the square root of the diagonal terms of the covariance matrix Eq.(36). This leads to

$$
\forall i \in\{1, \ldots, N\}, \quad \sigma_{\lambda_{i}}^{p}=\sigma_{f} \times \sqrt{\left[\boldsymbol{A}\left(\boldsymbol{I}+\boldsymbol{P} \boldsymbol{P}^{T}\right) \boldsymbol{A}^{T}\right]_{i i}}
$$

In the particular case of Finite Element (FE)-based DIC (e.g., [3]), the DoF correspond to the components of the nodal displacements. As a consequence, each $\sigma_{\lambda_{i}}^{p}$ directly matches the standard deviation of one component of the displacement at the location of the node related to $\lambda_{i}$

It is important to note that the standard deviation given by Eq. (37) depends on the derivatives of the gradient of image $\tilde{g}$ at non-integer locations, cf Eq.(26). We shall see in Section 3 that it is possible to estimate this quantity via linear interpolation of the gradient $\underline{\nabla}(\tilde{g})$. Another possibility is to approximate the Jacobian calculation using the gradient of $(f)$ at pixel $\underline{x}_{\mathrm{p}}$ instead of gradient of $(g)$ at pixel $\underline{x}_{\mathrm{p}}+\underline{u}\left(\underline{x}_{\mathrm{p}}, \boldsymbol{\lambda}\right)$. Approximated standard deviation $\bar{\sigma}_{\lambda_{i}}^{p}$ writes:

$$
\forall i \in\{1, \ldots, N\}, \quad \bar{\sigma}_{\lambda_{i}}^{p}=\sigma_{f} \times \sqrt{\left[\overline{\boldsymbol{A}}\left(\boldsymbol{I}+\boldsymbol{P} \boldsymbol{P}^{T}\right) \overline{\boldsymbol{A}}^{T}\right]_{i i}} \text { with } \overline{\boldsymbol{A}}=\boldsymbol{M}^{-1} \boldsymbol{L} .
$$

The following formula that can be found in the literature (e.g., $[17,8])$ to estimate the resolution. It does not take into account the sub-pixel displacement, and writes as follows:

$$
\forall i \in\{1, \ldots, N\}, \quad \hat{\sigma}_{\lambda_{i}}^{p}=\sigma_{f} \times \sqrt{2\left[\boldsymbol{M}^{-1}\right]_{i i}}
$$

This corresponds to the case where $\boldsymbol{P}$ is simply the identity matrix in Eq.(39). In this case, with the definition of matrix $\overline{\boldsymbol{A}}$, one obtains indeed $\overline{\boldsymbol{A}} \overline{\boldsymbol{A}}^{T}=\boldsymbol{M}^{-1} \boldsymbol{L}^{T}\left(\boldsymbol{M}^{-1} \boldsymbol{L}^{T}\right)^{T}=\boldsymbol{M}^{-1} \boldsymbol{L}^{T} \boldsymbol{L} \boldsymbol{M}^{-T}=$ $\boldsymbol{M}^{-1} \boldsymbol{M} \boldsymbol{M}^{-1}=\boldsymbol{M}^{-1}$. The proposed formula is thus a generalization of the formula which was previously available in the literature. Next section aims at comparing the simplified new prediction in Eq.(39) and the usual one in Eq.(40), assuming displacement is a mere rigid translation. 


\subsection{Particular case of a pure translation}

In the case of a sub-pixel rigid displacement along the $\underline{e}_{1}$ direction, matrix $\boldsymbol{P}$ defined in Eq.(19) writes as follows:

$$
\boldsymbol{P}=\left[\begin{array}{ccccc}
1-\alpha & \alpha & 0 & \ldots & 0 \\
0 & 1-\alpha & \alpha & \ddots & \vdots \\
\vdots & \ddots & \ddots & \ddots & 0 \\
0 & \ldots & 0 & 1-\alpha & \alpha
\end{array}\right]
$$

We assume here that the pixels are listed along the first axis, and then along the second axis. $\alpha$ lies between 0 and $1, \alpha=0$ corresponding to the absence of any displacement, and $\alpha=1$ corresponding to a 1-pixel displacement.

Let $a_{i j}$ denote the $(i, j)$ component of matrix $\overline{\boldsymbol{A}}$, i.e., $a_{i j}=[\overline{\boldsymbol{A}}]_{i j}$. Eq.(38) writes, for any $i \in\{1, \ldots, N\}$ :

$$
\begin{aligned}
\bar{\sigma}_{\lambda_{i}}^{p} & =\sigma_{f} \times \sqrt{\left[\overline{\boldsymbol{A}} \overline{\boldsymbol{A}}^{T}+\overline{\left.\boldsymbol{A} P \boldsymbol{P}^{T} \overline{\boldsymbol{A}}^{T}\right]_{i i}}\right.} \\
& =\sigma_{f} \times \sqrt{\sum_{k=1}^{N_{p}} a_{i k}^{2}+\sum_{k=1}^{N_{p}}\left((1-\alpha) a_{i k}+\alpha a_{i(k-1)}\right)^{2}}
\end{aligned}
$$

Eq.(40) gives

$$
\hat{\sigma}_{\lambda_{i}}^{p}=\sigma_{f} \times \sqrt{\sum_{k=1}^{N_{p}} a_{i k}^{2} .}
$$

The ratio between the proposed prediction of Eq.(38) and the one given in Eq.(40) is:

$$
\begin{aligned}
\forall i \in\{1, \ldots, N\}, \quad \frac{\bar{\sigma}_{\lambda_{i}}^{p}}{\hat{\sigma}_{\lambda_{i}}^{p}} & =\sqrt{\frac{1}{2}\left(1+\frac{\sum_{k=1}^{N_{p}}\left((1-\alpha) a_{i k}+\alpha a_{i(k-1)}\right)^{2}}{\sum_{k=1}^{N_{p}} a_{i k}^{2}}\right)} \\
& =\sqrt{\frac{1}{2}\left(1+\alpha^{2}+(1-\alpha)^{2}+2 \alpha(1-\alpha) \frac{\sum_{k=1}^{N_{p}} a_{i k} a_{i(k-1)}}{\sum_{k=1}^{N_{p}} a_{i k}^{2}}\right)} .
\end{aligned}
$$

$\left(\bar{\sigma}_{\lambda_{i}}^{p} / \hat{\sigma}_{\lambda_{i}}^{p}\right)^{2}$ has a quadratic dependency on $\alpha$. It can be checked that it reaches its maximum value equal to 1 for $\alpha=0$ or $\alpha=1$. This means that a translation of an integer pixel gives the same result in Eq. (38) and (40), as expected. The minimum value of $\left(\bar{\sigma}_{\lambda_{i}}^{p} / \hat{\sigma}_{\lambda_{i}}^{p}\right)^{2}$ equals to

$$
\frac{3}{4}+\frac{1}{4} \frac{\sum_{k=1}^{N_{p}} a_{i k} a_{i(k-1)}}{\sum_{k=1}^{N_{p}} a_{i k}^{2}}
$$

for $\alpha=1 / 2$.

As a sanity check, note that, from Cauchy-Schwarz inequality, $\left|\sum_{k=1}^{N_{p}} a_{i k} a_{i(k-1)}\right| \leq \sum_{k=1}^{N_{p}} a_{i k}^{2}$. Consequently, the minimum value is above $1 / 2$ and below 1 . This means that the formula given in Eq.(40) is a systematic overestimation of the value obtained when taking the interpolation into account. 


\subsection{Displacement resolution at any pixel}

The predictive formula of Eq.(38) only corresponds to the displacement resolution for FE-based DIC. The idea here is to generalize the proposed formula to any G-DIC (i.e., not only FE-based ones) and to extend it to the scale of the pixel. So one would have both the displacement and its resolution defined pixelwise, for any choice of the kinematics space $\mathcal{U}$.

Thanks to the matrix notation, function family $\left(\underline{\varphi}_{i}\right)_{1 \leq i \leq N}$ can also be written in two $N_{p} \times N$ matrices $\varphi_{\underline{e}_{1}}$ and $\varphi_{\underline{e}_{2}}$ such that once the convergence criterion is reached, we have

$$
\forall \underline{x}_{\mathrm{i}} \in \mathrm{RoI}, \quad \underline{u}\left(\underline{x}_{\mathrm{i}}, \tilde{\boldsymbol{\lambda}}^{\mathrm{opti}}\right)=\left[\boldsymbol{u}_{1}\right]_{i} \underline{e}_{1}+\left[\boldsymbol{u}_{2}\right]_{i} \underline{e}_{2} \quad \text { and } \quad\left\{\begin{array}{l}
\boldsymbol{u}_{1}=\boldsymbol{\varphi}_{\underline{e}_{1}} \tilde{\boldsymbol{\lambda}}^{\mathrm{opti}} \\
\boldsymbol{u}_{2}=\boldsymbol{\varphi}_{\underline{e}_{2}} \tilde{\boldsymbol{\lambda}}^{\mathrm{opti}}
\end{array} .\right.
$$

$\boldsymbol{u}_{1}$ (resp. $\boldsymbol{u}_{2}$ ) corresponds to the vector made of the displacements at each pixel location along direction $\underline{e}_{1}$ (resp. $\underline{e}_{2}$ ), associated with the affected optimized DoF vector $\tilde{\boldsymbol{\lambda}}^{\text {opti }}$. According to Prop.(2.1), $\boldsymbol{u}_{1}$ and $\boldsymbol{u}_{2}$ satisfy:

$$
\left\{\begin{array}{c}
\text { mean : }\left\langle\boldsymbol{u}_{1,2}\right\rangle=\boldsymbol{\varphi}_{\underline{e}_{1,2}}\langle\delta \boldsymbol{\lambda}\rangle \\
=\boldsymbol{\varphi}_{\underline{e}_{1,2}} \boldsymbol{A}(\boldsymbol{f}+\boldsymbol{P} \boldsymbol{g}) \\
\text { covariance matrix : } \boldsymbol{\Sigma}_{\boldsymbol{u}_{1,2}}=\boldsymbol{\varphi}_{\underline{e}_{1,2}} \boldsymbol{\Sigma}_{\delta \boldsymbol{\lambda}} \boldsymbol{\varphi}_{\underline{e}_{1,2}^{T}}^{T} \\
\quad=\sigma_{f}^{2} \times \boldsymbol{\varphi}_{\underline{e}_{1,2}} \boldsymbol{A}\left(\boldsymbol{I}+\boldsymbol{P} \boldsymbol{P}^{T}\right) \boldsymbol{A}^{T} \boldsymbol{\varphi}_{\underline{e}_{1,2}}^{T}
\end{array}\right.
$$

The standard deviation $\sigma_{u, 1}^{p}\left(\underline{x}_{\mathrm{i}}\right)$ (resp. $\sigma_{u, 2}^{p}\left(\underline{x}_{\mathrm{i}}\right)$ ) of the first (resp. second) component of the displacement at pixel $\underline{x}_{\mathrm{i}}$ can thus be calculated as the square root of the diagonal terms of the covariance matrix defined in Eq.(49). This leads to

$$
\forall \underline{x}_{\mathrm{i}} \in \operatorname{RoI}, \quad \sigma_{u, \ell}^{p}\left(\underline{x}_{\mathrm{i}}\right)=\sigma_{f} \times \sqrt{\left[\boldsymbol{\varphi}_{\underline{e}_{\ell}} \boldsymbol{A}\left(\boldsymbol{I}+\boldsymbol{P} \boldsymbol{P}^{T}\right) \boldsymbol{A}^{T} \boldsymbol{\varphi}_{\underline{e}_{\ell}}^{T}\right]_{i i}} .
$$

\subsection{Conclusion}

This section recalled the DIC basics by putting the emphasis on the role of interpolation. A generalized predictive formula for DIC DoF resolution, which takes into account interpolation, was therefore demonstrated. Finally, this predictive formula is extended to the resolution of DIC displacement. This leads indeed to a pixelwise prediction of the resolution. Next section focuses on the numerical validation of Eq.(38) and Eq.(50) .

\section{Validation with synthetic data}

To illustrate the difference between the results obtained with equations Eq.(38), Eq.(39), and Eq.(40), we consider two synthetic datasets. The purpose of this section is to validate the proposed formula Eq.(50) under the Gaussian white noise assumption. In real experiments, the validation of this formula is more complex. For instance, noise of usual camera sensor is actually not a white noise as assumed in Hyp.(1). In addition, rigid body motion generally occurs during testing. These issues are the purpose of studies concerning another measurement technique $[23,10]$. They are out of the scope of this paper, but directly concern [4], where the present improved predictive formula is experimentally verified. This section is organized as follows. A synthetic dataset is first detailed and notations are introduced. The pixelwise prediction of the resolution is then highlighted and the formula is finally validated using these synthetic datasets. 


\subsection{Synthetic data}

In order to properly define a consistent set of synthetic data, $N_{\text {img }}$ pairs of reference $\left(f_{t}\right)_{1 \leq t \leq N_{\text {img }}}$ and current $\left(g_{t}\right)_{1 \leq t \leq N_{\mathrm{img}}}$ images are simulated. These images are built up from a pair of noiseless images $\left(f_{0}, g_{0}\right)$. To ensure the random aspect of the pattern digitized in the images, it is defined from a real image $(h)$ depicted in Fig.(1). To limit interpolation errors, which may occur during the elaboration of the current image, an overkill pair of images $(F, G)$ are defined. The process is detailed below:

$i)$ image $(h)$ is interpolated on a four-time finer discrete pixel map than the one required for $\left(f_{0}\right)$ and $\left(g_{0}\right)$, defining image $(F)$;

ii) the gray value of each pixel of image $\left(f_{0}\right)$ is computed by averaging the gray values of pixels of $(F)$ included in each pixel of $(f)$;

iii) image $F$ is deformed accounting for a prescribed displacement $\underline{U}$, leading to $G$;

$i v$ ) the gray value of each pixel of image $\left(g_{0}\right)$ is computed by averaging the gray values at pixels of $(G)$ included in each pixel of $(g)$.

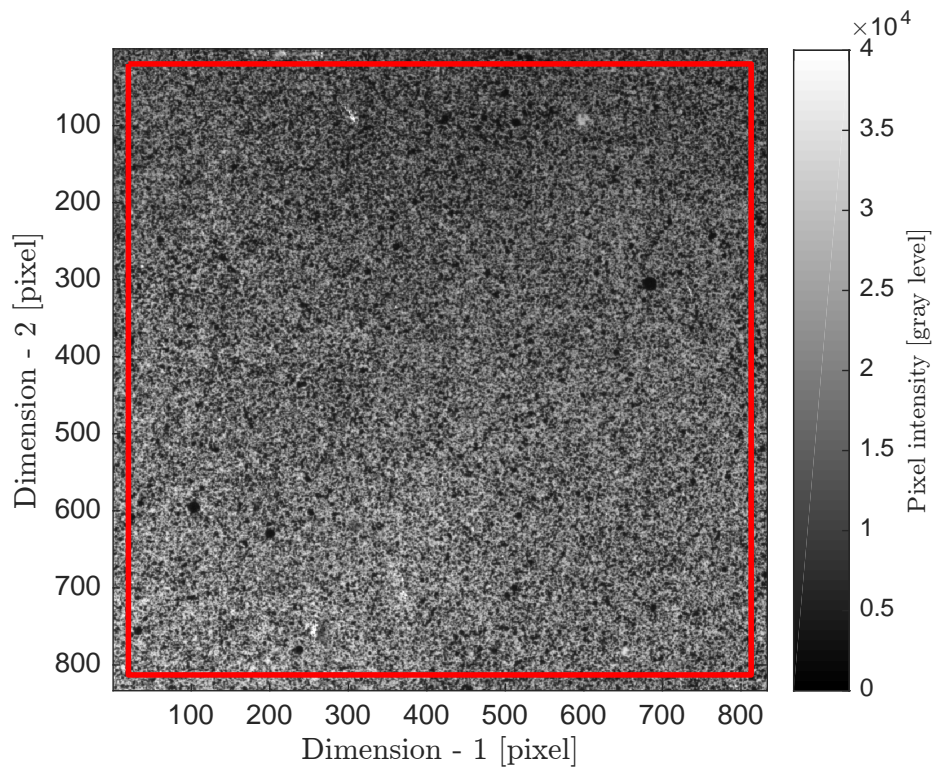

Figure 1: Real speckle $(h)$

Then, Gaussian white noises of 0 -mean and standard deviation $\sigma_{f}$ are added independently to

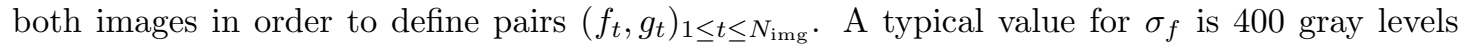
in usual 16-bit images. This value corresponds to a typical order of magnitude of the standard deviation of the noise reported in the literature (e.g., $[8,4])$.

Remark 3.1 We also implemented a eight-time finer separation between images $(F, G)$ and $f_{0}, g_{0}$. Obtained results were identical, so we consider that the consequence of image noise is greater than interpolation artefacts which are introduced during image generation. 
$N_{\text {img }}$ DIC procedures are applied afterwards on these pairs of images to recover the related displacement fields $\underline{u}_{t}$. Thanks to these calculations, an empirical displacement resolution is calculated, such that

$$
\sigma_{u, \ell}^{e}\left(\underline{x}_{\mathrm{p}}\right)=\sqrt{\frac{1}{N_{\mathrm{img}}-1} \sum_{t=1}^{N_{\mathrm{img}}}\left(u_{t, \ell}\left(\underline{x}_{\mathrm{p}}\right)-\left\langle u_{\ell}\left(\underline{x}_{\mathrm{p}}\right)\right\rangle\right)^{2}}
$$

with

$$
\left\langle u_{\ell}\left(\underline{x}_{\mathrm{p}}\right)\right\rangle=\frac{1}{N_{\mathrm{img}}} \sum_{t=1}^{N_{\mathrm{img}}} u_{\ell}\left(\underline{x}_{\mathrm{p}}\right)_{t} .
$$

Since the usual prediction only offers the resolution of the DoF, it gives the resolution at the nodal displacements in the case of FE-based DIC. Here, the nodes is defined in such a way that their coordinates directly correspond to the pixel locations. This allows the calculation of an empirical DoF resolution. For any $i \in\{1, \ldots, N\}$

$$
\sigma_{\lambda_{i}}^{e}=\sigma_{u, \ell}^{e}\left(\underline{x}_{k}\right)
$$

where $\underline{x}_{k}$ and $\ell$ respectively correspond to the pixel location of the node and the component of the displacement related to $\mathrm{DoF} \lambda_{i}$.

Moreover, it is worth noting that in order to verify Hyp.(3), the DIC tangent operator $\boldsymbol{M}$ must be elaborated with the noiseless reference image. In practice, the gradient of the noiseless image can be approximated by the gradient of the average reference image $\left\langle f_{t}\right\rangle$ defined as the mean of reference images $\left(f_{t}\right)_{1 \leq t \leq N_{\mathrm{img}}}$. This gives $\boldsymbol{M}_{\langle t\rangle}$ for any image:

$$
\boldsymbol{M}_{\langle t\rangle}=\boldsymbol{L}_{\langle t\rangle}^{T} . \boldsymbol{L}_{\langle t\rangle} \quad \text { with }\left\{\begin{array}{l}
{\left[\boldsymbol{L}_{\langle t\rangle}\right]_{i j}=\underline{\nabla}\left\langle f_{t}\left(\underline{x}_{\mathrm{i}}\right)\right\rangle \cdot \underline{\varphi}_{j}\left(\underline{x}_{\mathrm{i}}\right)} \\
\left\langle f_{t}\right\rangle=\frac{1}{N_{\mathrm{img}}} \sum_{t=1}^{N_{\mathrm{img}}} f_{t}
\end{array} .\right.
$$

With this noise-free tangent operator, the new resolutions of Eq.(38), Eq.(39) and Eq.(50) and the one given in Eq.(40) respectively write:

$$
\begin{array}{ll}
\forall \underline{x}_{\mathrm{i}} \in \mathrm{RoI}, \quad & \sigma_{u, \ell}^{p}\left(\underline{x}_{\mathrm{i}}\right)=\sigma_{f} \times \sqrt{\left[\boldsymbol{\varphi}_{\underline{e}_{\ell}} \cdot \boldsymbol{A}_{\langle t\rangle} \cdot\left(\boldsymbol{I}+\boldsymbol{P} \cdot \boldsymbol{P}^{T}\right) \cdot \boldsymbol{A}_{\langle t\rangle}^{T} \cdot \boldsymbol{\varphi}_{\underline{e}_{\ell}}^{T}\right]_{i i}} ; \\
\forall i \in\{1, \ldots, N\}, \quad & \sigma_{\lambda_{i}}^{p}=\sigma_{f} \times \sqrt{\left[\boldsymbol{A}_{\langle t\rangle} \cdot\left(\boldsymbol{I}+\boldsymbol{P} \cdot \boldsymbol{P}^{T}\right) \cdot \boldsymbol{A}_{\langle t\rangle}^{T}\right]_{i i}} \\
& \bar{\sigma}_{\lambda_{i}}^{p}=\sigma_{f} \times \sqrt{\left[\overline{\boldsymbol{A}}\langle t\rangle \cdot\left(\boldsymbol{I}+\boldsymbol{P} \cdot \boldsymbol{P}^{T}\right) \cdot \overline{\boldsymbol{A}}_{\langle t\rangle}^{T}\right]_{i i}}, \\
& \hat{\sigma}_{\lambda_{i}}^{p}=\sigma_{f} \times \sqrt{2\left[\boldsymbol{M}_{\langle t\rangle}^{-1}\right]_{i i}} .
\end{array}
$$

with

$$
\boldsymbol{A}_{\langle t\rangle}=-\boldsymbol{M}_{\langle t\rangle}^{-1} \cdot \boldsymbol{J}_{\tilde{\boldsymbol{r}}}^{T} \text {, and } \overline{\boldsymbol{A}}_{\langle t\rangle}=\boldsymbol{M}_{\langle t\rangle}^{-1} \cdot \boldsymbol{L}_{\langle t\rangle}
$$

and where the Jacobian $\boldsymbol{J}_{\tilde{\boldsymbol{r}}}$ is calculated using only one of the DIC solutions. It is here arbitrarily the first one.

The DIC tangent operator $\boldsymbol{M}$ is usually elaborated using only one image $\left(\langle f\rangle_{t}\right)$. In order to highlight the improvement that offers the use of a noise-free tangent operator, $N_{\text {img }}$ classic DIC were also performed on the pairs $\left(f_{t}, g_{t}\right)_{1 \leq t \leq N_{f}}$. For each solving however, the tangent operator $M$ was built up using the gradient of image $\left(f_{t}\right)$. Let $\left(\hat{\hat{u}}_{t}\right)_{1 \leq t \leq N_{\text {img }}}$ be the displacements output of 


\begin{tabular}{|c|c|c|c|}
\hline Ratio & Definition & $\begin{array}{c}\text { Image gradient used for the elaboration } \\
\text { of DIC tangent operator } \boldsymbol{M}\end{array}$ & $\begin{array}{c}\text { Formula implemented for } \\
\text { prediction computation }\end{array}$ \\
\hline$\hat{\rho}_{\lambda_{i}}$ & $\hat{\sigma}_{\lambda_{i}}^{e} \hat{\hat{\sigma}}_{\lambda_{i}}^{p}$ & $\underline{\nabla}\left(f_{t}\right)$ (noisy) & "usual" (cf Eq.(60)) \\
\hline$\hat{\rho}_{\lambda_{i}}$ & $\sigma_{\lambda_{i}}^{e} \hat{\sigma}_{\lambda_{i}}^{p}$ & $\underline{\nabla}\left(\left\langle f_{t}\right\rangle\right)$ (noise free, $c f$ Eq.(54)) & "usual" (cf Eq.(58)) \\
\hline $\bar{\rho}_{\lambda_{i}}$ & $\sigma_{\lambda_{i}}^{e} / \bar{\sigma}_{\lambda_{i}}^{p}$ & $\underline{\nabla}\left(\left\langle f_{t}\right\rangle\right)$ (noise free, $c f$ Eq.(54)) & generalized $(c f$ Eq.(57)) \\
\hline$\rho_{\lambda_{i}}$ & $\sigma_{\lambda_{i}}^{e} / \sigma_{\lambda_{i}}^{p}$ & $\underline{\nabla}\left(\left\langle f_{t}\right\rangle\right)$ (noise free, $c f$ Eq.(54)) & generalized $(c f$ Eq.(56)) \\
\hline$\rho_{u, \ell}$ & $\sigma_{u, \ell}^{e} / \sigma_{u, \ell}^{p}$ & $\underline{\nabla}\left(\left\langle f_{t}\right\rangle\right)$ (noise free, $c f$ Eq.(54)) & generalized $(c f$ Eq.(55)) \\
\hline
\end{tabular}

Table 1: Ratios between empirical and predicted resolution with their features.

these $N_{\mathrm{img}}$ DIC. In a similar route as for $\hat{\sigma}_{\lambda_{i}}^{p}$ and $\sigma_{\lambda_{i}}^{e}$, both the prediction $\hat{\hat{\sigma}}_{\lambda_{i}}^{p}$ and the calculation of the empirical resolution $\hat{\hat{\sigma}}_{\lambda_{i}}^{e}$ are elaborated, such that:

$$
\begin{aligned}
\forall i \in\{1, \ldots, N\}, \quad \hat{\hat{\sigma}}_{\lambda_{i}}^{p}=\sigma_{f} \times \sqrt{2\left[\boldsymbol{M}^{-1}\right]_{i i}}, \\
\hat{\hat{\sigma}}_{\lambda_{i}}^{e}=\hat{\hat{\sigma}}_{\hat{\hat{u}}, \ell}^{e}\left(\underline{x}_{k}\right)=\sqrt{\frac{1}{N_{\mathrm{img}}-1} \sum_{t=1}^{N_{\text {img }}}\left\{\left(\hat{\hat{u}}_{t, \ell}\left(\underline{x}_{\mathrm{p}}\right)-\left\langle\hat{\hat{u}}_{\ell}\left(\underline{x}_{\mathrm{p}}\right)\right\rangle\right)^{2}\right\} .}
\end{aligned}
$$

The tangent operator $\boldsymbol{M}$, which is used in the prediction resolution Eq.(60), is one of the $N_{\mathrm{img}}$ DIC made for computing $\left(\hat{\hat{u}}_{t}\right)_{1 \leq t \leq N_{\mathrm{img}}}$. It is here arbitrarily the first one.

Finally, five ratios between prediction and observation of the resolution are defined. They highlight the different features of both the predictive formulas and the improvement that can offer the noiseless definition of the DIC tangent operator. These ratios are defined as follows

$$
\forall \lambda_{i} \in\left(\lambda_{i}\right)_{1 \leq i \leq N}, \hat{\hat{\rho}}_{\lambda_{i}}=\frac{\hat{\hat{\sigma}}_{\lambda_{i}}^{e}}{\hat{\hat{\sigma}}_{\lambda_{i}}^{p}}, \hat{\rho}_{\lambda_{i}}=\frac{\hat{\sigma}_{\lambda_{i}}^{e}}{\sigma_{\lambda_{i}}^{p}}, \bar{\rho}_{\lambda_{i}}=\frac{\sigma_{\lambda_{i}}^{e}}{\bar{\sigma}_{\lambda_{i}}^{p}}, \rho_{\lambda_{i}}=\frac{\sigma_{\lambda_{i}}^{e}}{\sigma_{\lambda_{i}}^{p}} \text { and } \forall \ell \in\{1,2\} \rho_{u, \ell}=\frac{\sigma_{u, \ell}^{e}}{\sigma_{u, \ell}^{p}} .
$$

The characteristics of each ratio is summarized in Tab.(1). Results are presented in the next section. While the 1-D case is a preliminary validation with a simplified uniform displacement (Section 3.2), the 2-D case allows us to discuss the proposed prediction formula under a nonuniform displacement (Section 3.3).

\subsection{1-D uniform displacement}

A simple 1-D case is implemented here for a first validation of the predictive formula. Indeed, the low computing cost of a 1-D DIC procedure permits to perform it on a large number of images; $N_{\text {img }}$ equals here $10^{4}$. Such a number enables a good estimation of the empirical standard deviation. The 1-D speckle is here elaborated from a line of the real image $(h)$ depicted in Fig.(1). Fig.(2) illustrates the noiseless reference image $\left(f_{0}\right)$. The prescribed displacement $\underline{U}$ applied to image $\left(g_{0}\right)$ consists of a uniform translation of magnitude $\alpha$, such that $\alpha \in[0,1]$. The DIC kinematics are defined through a unique DoF denoted $\lambda$. It is the 1-D rigid-body motion of the RoI, which is set here to the 40-pixel area in the middle of the image. Finally, we considered here the only case for which the DIC tangent operator is defined using the noiseless reference image $\left(\langle f\rangle_{t}\right)$, and the interpolation required within the DIC minimization is the linear one.

The $N_{\text {img }}$ DIC calculations allow the calculation of the empirical $\sigma_{\lambda}^{e}$ with Eq.(51), while the predictive formulas are applied to elaborate the usual prediction $\hat{\sigma}_{\lambda}^{p}$ from Eq.(58), the generalized 


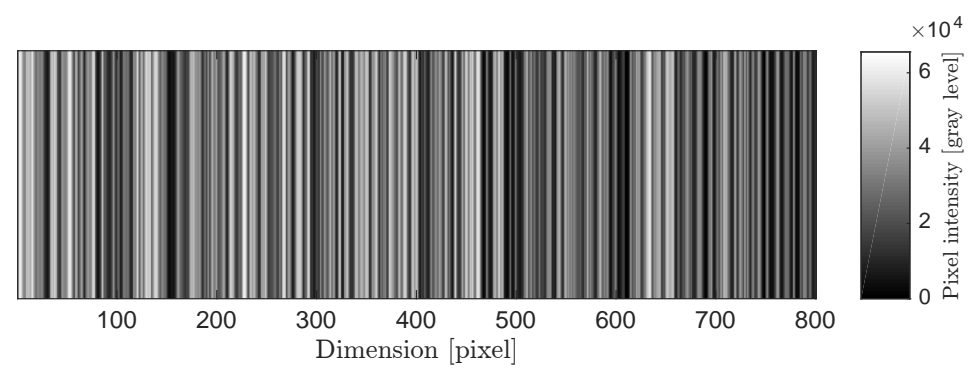

Figure 2: Illustration of the line, which is defined from one line of $(h)$, used for the 1-D study.

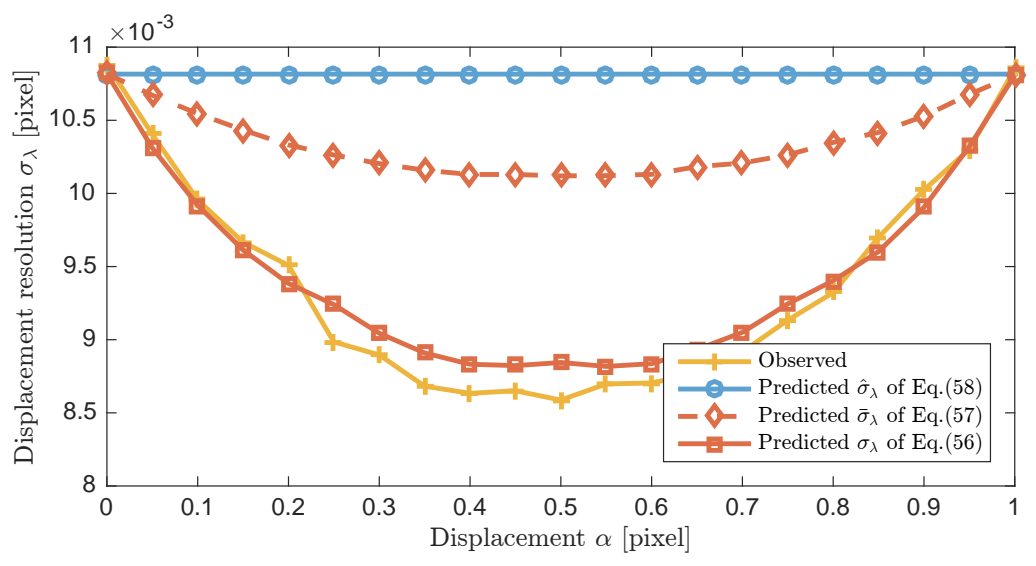

Figure 3: Comparison between the resolution predicted with different formulas and the empirical one.

one $\sigma_{\lambda}^{p}$ from Eq.(56) and its approximated version $\bar{\sigma}_{\lambda}^{p}$ from Eq.(57). This analysis is repeated 21 times, using several values for $\alpha$. From this data, the evolution of resolution $\sigma_{\lambda_{i}}^{e}$ with respect to sub-pixel displacement (i.e., $\alpha$ ) is observed. Results are illustrated in Fig.(3). The three types of predictions $\hat{\sigma}_{\lambda}^{p}$ (with Eq.(58)), $\bar{\sigma}_{\lambda}^{p}$ (with Eq.(57)) and $\sigma_{\lambda}^{p}$ (with Eq.(56)) are also shown. Of course, the prediction $\hat{\sigma}_{\lambda}^{p}$ does not depend on sub-pixel displacement. The prediction value is thus constant for all tested $\alpha$. On the contrary, the proposed predictions $\bar{\sigma}_{\lambda}^{p}$ and $\sigma_{\lambda}^{p}$ take into account the sub-pixel part of the displacement. The decrease in resolution for the sub-pixel displacement is indeed perceptible in observations and also in the proposed predictions. Using the prediction of Eq.(58) leads to an over estimation of $20 \%$ for a sub-pixel displacement equals to 0.5 .

It is possible to note that the observed parabolic prediction is in agreement with the discussion of Section 2.3. Moreover, the prediction $\sigma_{\lambda}^{p}$, which is evaluated based on a linear interpolation of the Jacobian $\boldsymbol{J}_{\tilde{r}}$, outperforms the other approaches. The prediction $\bar{\sigma}_{\lambda}^{p}$, which approximates $\boldsymbol{J}_{\tilde{r}}$ by $-L$, leads to an overestimation of the displacement resolution.

After this 1-D validation with a uniform displacement, we discuss now a more general 2-D 
case with a non-uniform displacement field.

\section{$3.3 \quad 2-D$ non-uniform displacement}

The full image $(h)$ is used now, and images $\left(f_{0}\right)$ and $\left(g_{0}\right)$ are built with a size of $832 \times 832\left[\mathrm{pixel}^{2}\right]$. The displacement $\underline{U}$ is defined as a rotation by an angle of $0.5^{\circ}$ around the image center. Over the Region of Interest (the whole image except a border of 20 pixels), the displacement norm lies between 0 and 3 [pixel]. The effect of the sub-pixel displacement can thus be easily observed, the displacement gently evolving between successive integer values for the displacement in pixel. The FE-DIC implemented is defined here through regular quad elements of size $13 \times 13$ [pixel $^{2}$ ], with four nodes. This arbitrary choice is made in order to have enough nodes along each direction. There are indeed here $62 \times 62$ nodes, and kinematics is thus defined through $N=7688 \mathrm{DoF}$. Nevertheless, elements constituting the border of the RoI have been excluded of the statistical analysis because border nodes are not linked to four quads as interior ones are. Finally, because of the relative cost required by each calculation, $N_{\mathrm{img}}$ is reduced to 200 here. This certainly induces an additional error compared to the 1-case where $N_{\text {img }}=10^{4}$ was used.

Two kinds of results are presented: Section 3.2.1 focuses on the pixel scale resolution; Section 3.2.2 discusses the sub-pixel interpolation effect and compares the different predictions with the observation.

\subsubsection{Pixelwise resolution features}

To illustrate the pixelwise calculation of the generalized predictive formula, let us first focus on a unique element. Fig.(4a-b) illustrate both close-ups of the typical prediction $\sigma_{u, 2}^{p}$ and observation $\sigma_{u, 2}^{e}$ of the vertical displacement resolution at an element scale (in particular in element 1700). The FE kinematics used within the DIC procedure is defined on four-nodes quads, which have a bilinear element-wise kinematics; and $\boldsymbol{\varphi}_{\underline{e}_{1,2}}$ also contain this bilinear feature. Finally, the predictive formula of Eq.(55) transforms this bilinear behavior into a biquadratic one. This behavior is clearly visible in both close-ups of the prediction and observation of the vertical resolution in Fig.(4a-b). Moreover, the prediction is in good agreement with the observation, as illustrated in Fig.(4c). The ratio $\rho_{u, 2}$ between both resolutions indeed slightly fluctuates around 1.08 and is almost constant for all elements. These results are not only denoting the behavior in element 1700: Fig.(4d) represents the average value that the ratio reaches, when computed within each element of the mesh. This value is again spatially equally distributed and is approximately equal to 1.08 .

Both the theory and the experiment allow thus to state that the displacement resolution is lower in the middle of an element. Nevertheless this improvement in term of resolution has to be counterbalanced by the impairment of spatial resolution. As depicted in Fig.(5), the displacement calculation at a pixel located just below a node uses pixels located in the 4 elements surrounding this node, whereas the same calculation at a pixel in the middle of an element relies in the 4 nodes of this element, and thus the pixels embedded in the 9 elements supporting these nodes. The area of pixels concerned by the displacement calculation at pixel location, i.e., the spatial resolution, is thus not constant. For instance, for pixels which are exactly located at nodal coordinates, this area is less than the half of this one needed for pixels in the middle of elements.

These first results illustrate the pixelwise feature of the generalized prediction introduced here. The prediction satisfactorily corresponds to the measurements and the observable quadratic behavior of the resolution within an element is also correctly represented by the prediction. The next section focuses on the DoF resolution, and the generalized prediction is compared with the 


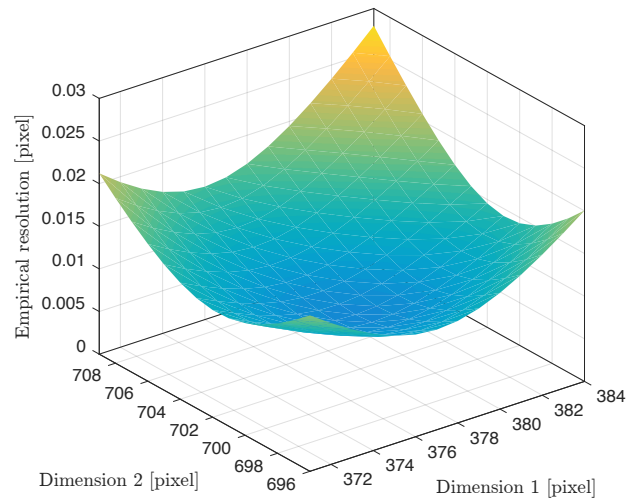

(a) Empirical resolution $\sigma_{u, 2}^{p}$ in element 1700

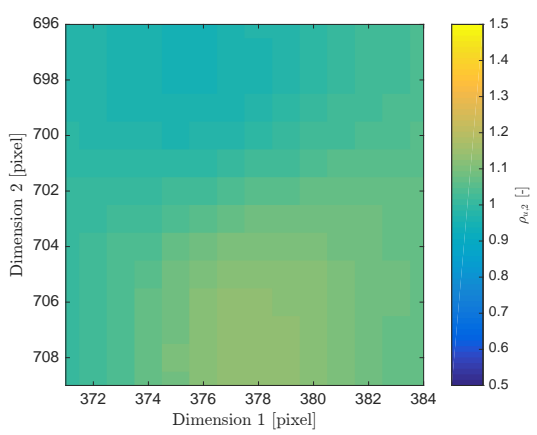

(c) Ratio $\rho_{u, 2}$ in element 1700

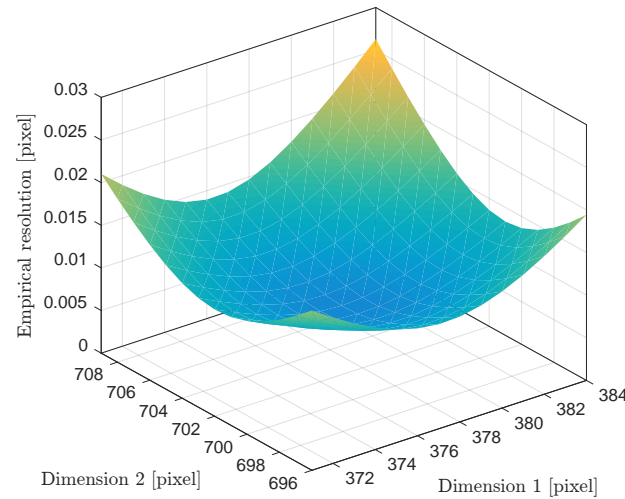

(b) Predicted resolution $\sigma_{u, 2}^{e}$ in element 1700

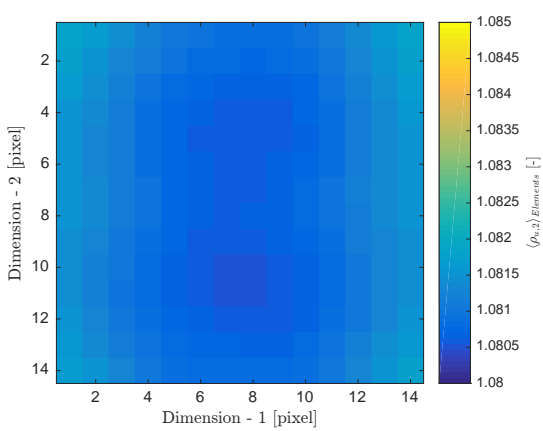

(d) Averaged ratio value over the elements

Figure 4: Observation (a), prediction (b) and their ratio (c) of the displacement resolution within element 1700. The same ratio can be computed for each elements and (d) maps its average. 


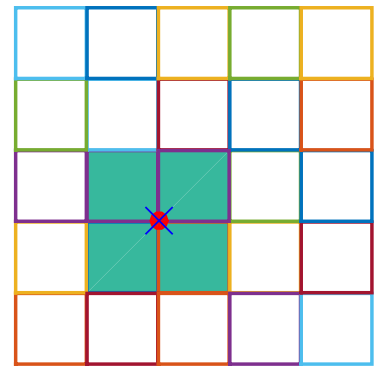

(a) Pixel below a node

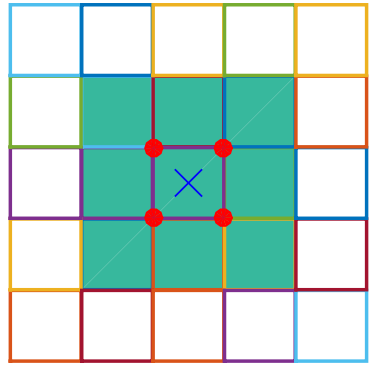

(b) Pixel at the middle of an element

Figure 5: Illustration of the supports associated with pixel displacement calculation at two extremal cases: (a) the pixel (cross) is located exactly below a node and (b) the pixel (cross) is located exactly at the middle of the element. Supports are colored in green. The squares represent the elements and the red circles illustrate the nodes.

usual one from the literature.

\subsubsection{Sub-pixel displacement and DoF resolution features}

This section focuses on DoF resolution, so that it is possible to compare directly the prediction o the literature given in Eq.(58) and the generalized predictions of the present paper. Results associated with both the bilinear and the bicubic interpolations are presented. The empirical estimations of the vertical displacement resolution $\sigma_{\lambda_{i}}^{e}$ are mapped in Fig.(6). Left- (resp. right) hand figure corresponds to the bilinear (resp. bicubic) interpolation. The vertical bands of highest values, which are observable in both resolution maps, correspond to the location where the sub-pixel part of the vertical displacement is close to 0 . In particular, the vertical displacement equals 0 in the middle band and respectively +1 and -1 for the right and left ones. The effect of the sub-pixel displacement on the displacement resolutions is thus clearly observable. Both interpolation schema provide however similar results. The order of magnitude of both resolutions is the same, especially for the highest values.

In this paper, the effect of such sub-displacements on resolution maps is theoretically associated with the interpolation required for the back-deformed image $\left(g_{\mathrm{BD}}\right)$ during DIC solving. It explains that both interpolations induce similar resolutions. Indeed, when the displacement corresponds to integer pixels, there is simply no interpolation. Since the highest values in the two maps of Fig.(6) correspond to integer displacement along direction $y$, they have the same numerical value, as expected from the theory.

More specifically, sensor noise is also interpolated and its statistical properties are deduced. The variance of the noise corrupting the back-deformed noise is thus defined in Eq.(37) as the diagonal terms of $\boldsymbol{\Sigma}_{n_{g_{\mathrm{BD}}}}=\sigma_{f}^{2} \times \boldsymbol{P} . \boldsymbol{P}^{T}$. The original noise variance is thus weighted by the diagonal terms of $\boldsymbol{P} . \boldsymbol{P}^{T}$. This quantity is depicted in Fig.(7) for both interpolation methods. As expected, this weighting term equals 1 where the sub-pixel displacement reaches 0 , and takes 

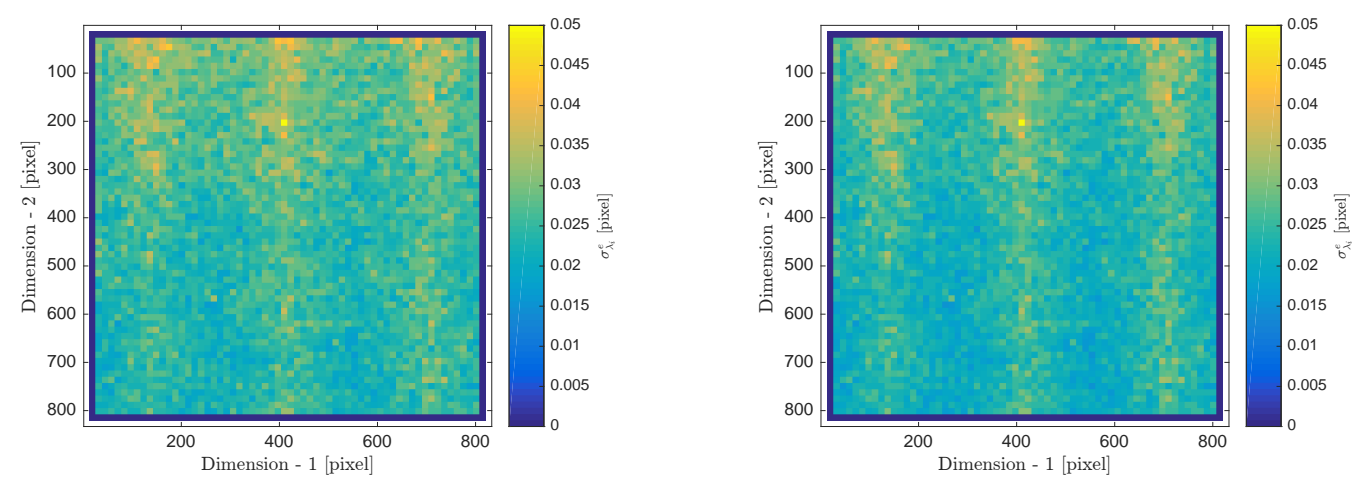

Figure 6: Empirical estimation of the standard deviation of the vertical displacement $\sigma_{\lambda_{i}}^{e}$. The two maps correspond to the two interpolations which have been implemented: the bilinear one (left) and the bicubic one (right).

lower values otherwise. The horizontal (resp. vertical) lines that are observed in left- and righthand sub-figures of Fig.(7) correspond to zero vertical (resp. horizontal) sub-pixel displacements. Only the pixels with zero sub-pixel displacement along both vertical and horizontal directions are affected by a noise identical to that of the reference image. Finally, we observe that the bicubic interpolation tends to have a smoother weighting effect. In Fig.(7), the lowest values are indeed lower in the left-hand side for an identical width. This can be easily explained with the 1-D case: at the critical sub-pixel displacement of 0.5 , the weighting term equals 0.5 for the linear interpolation while it reaches 0.64 for the cubic case. Consequently, the effect of the interpolation on the displacement resolution is slightly reduced for the cubic interpolation.

It is worth noting that in the literature, the noise corrupting the back-deformed image is usually assumed to be the same as the one corrupting the reference image, namely a Gaussian white noise. The obtained weighting maps are thus to be compared with a constant map of value 1.

In order to see how the new prediction $\sigma_{u, 2}^{p}$ is influenced by this weighting function, Fig.(8) plots the windowed means of the empirical and predicted values of the vertical displacement resolution with respect to the sub-pixel part of the displacement. The left- (resp. right-) hand side figure illustrates the results when the bilinear (resp. bicubic) interpolation is implemented.

As expected, the new prediction $\sigma_{u, 2}^{p}$ introduced here is sub-pixel-dependent and close to the observation for both interpolations. Note that the $\hat{\sigma}_{u, 2}^{p}$ would lead to a plateau taking the values reached for zeros sub-pixel displacements, whatever the implemented interpolation.

For the bilinear case illustrated in the left-hand side plot in Fig.(8), the prediction equals the observation for integer values of the horizontal sub-pixel displacement, i.e., for $u_{x, 1}=0$ or for 1. In-between, the prediction slightly depends on this horizontal sub-pixel part of the displacement. The prediction is indeed coupled with both the horizontal and vertical displacements. Such coupling is surprisingly not noticeable in the observations. There is consequently a little underestimation of the proposed prediction. A similar effect is observable for the bicubic interpolation, as depicted in the right-hand side plot in Fig.(8). Note that the true displacement between reference and current images is here a rotation. The DIC kinematics are based in this case on 2-D FE shape functions and thus cannot perfectly recover the sought displacement. This poor kinematics definition can explain the difference between the observed and the predicted 

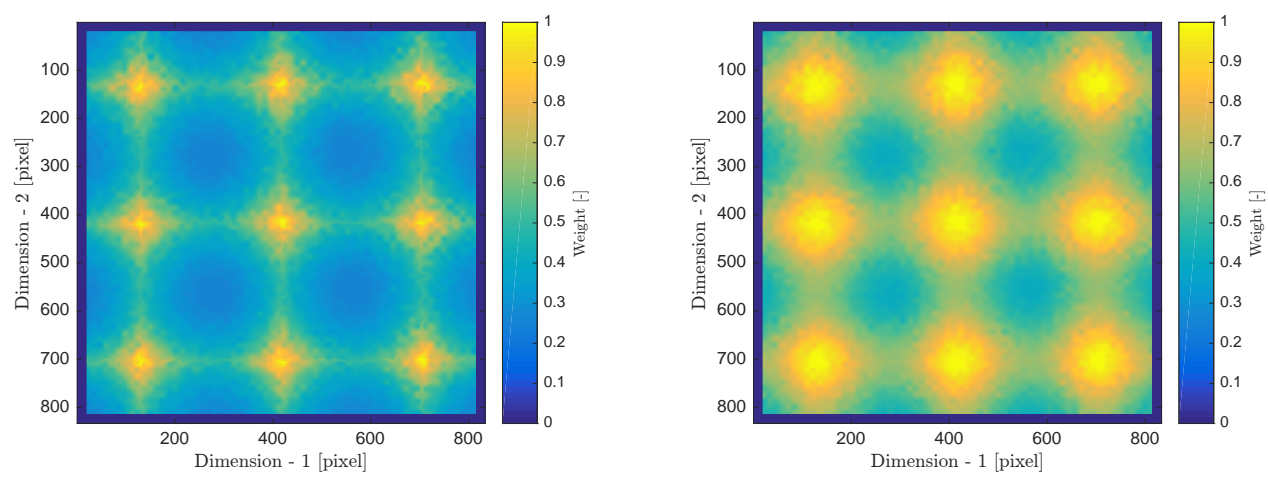

Figure 7: Map of the diagonal terms of $\boldsymbol{P} \cdot \boldsymbol{P}^{T}$. The two maps correspond to the two interpolations which have been implemented: the bilinear one (left) and the bicubic one (right).

values.

It is worth noting that the prediction is associated here with an optimized DIC process, since its tangent operator used is built up using noise-free image gradient $\underline{\nabla}\left(\left\langle f_{t}\right\rangle\right)$. So as to highlight the improvement of both the use of a noise-free tangent operator and the new prediction, ratios are now considered. Fig.(9) maps the four ratios $\hat{\hat{\rho}}_{\lambda}, \hat{\rho}_{\lambda}, \bar{\rho}_{\lambda}$ and $\rho_{\lambda}$ between observation and prediction of the vertical displacement resolution. Their respective definition is given in Tab.1.

The maps in Fig.(9a-c) present the maps associated with these ratios. The vertical bands of higher values, that are clearly visible in Fig.(9a), less in Fig.(9b) and even less in Fig.(9c), correspond to the effect of vertical sub-pixel displacement, which is better taken into account from $\hat{\hat{\rho}}_{u}$ to $\hat{\rho}_{u}$ and then to $\bar{\rho}_{u}$. These bands disappear in Fig.(9d), but others which are horizontal appears. They correspond to the location where the horizontal sub-pixel displacement is far from zero. Ratio $\rho_{u}$ indeed highlights such coupling effects.

The histograms in Fig.(9e-h) illustrate the same results, but the distribution properties are more quantifiable. On the one hand, the mean value of the ratio is closer to one from the usual predictions to the generalized one. On the other hand, the sharpness of the distribution is also improved. The formula from the literature gives indeed an over-estimation of about $15 \%$, with a distribution characterized by a standard deviation of 0.09 , which as to be compared with the new prediction that leads to an over-estimation of about $8 \%$, with a standard deviation characterizing its distribution of 0.07 . One can also remark that the ratio $\hat{\rho}_{u}$ already gives reliable estimation, thanks to the fact that its associated DIC tangent operator has been elaborated with the noisefree average images.

\section{Guidelines}

Before concluding, we propose to the reader interested in applying this prediction the following guidelines:

1. Take a stack of images of the reference state. These images will be used to

- Compute the standard deviation of the camera sensor noise. Please note that Hyp.(1) can be relaxed to allow a pixelwise definition of the noise standard deviation. 

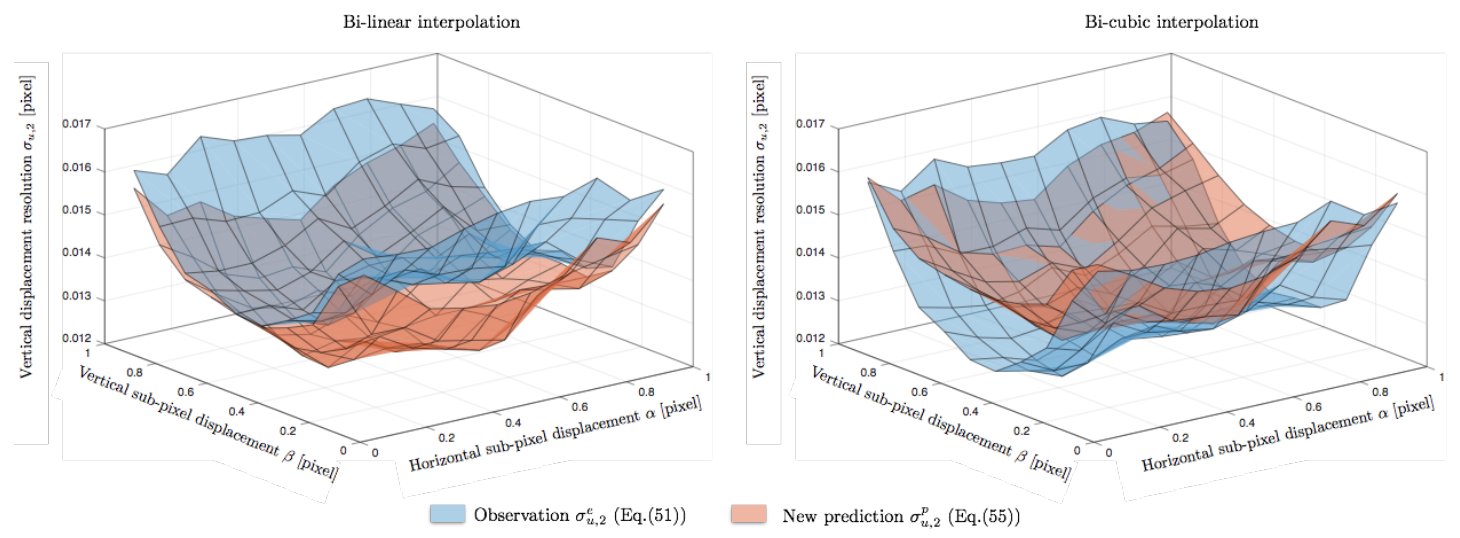

Figure 8: Observation and prediction of the standard deviation of the vertical displacement versus sub-pixel displacement. Continuous lines represent the respective average. The two plots correspond to the two interpolations which have been implemented: the bilinear one (left) and the bicubic (right) one.

- Build up a noise-free reference image, by averaging the reference images.

- Elaborate a noise-free DIC tangent operator, cf Eq.(54).

2. Shot the current image of the deformed specimen.

3. Apply DIC to retrieve the displacement field.

4. Calculate the Jacobian using the gradient of the noisy current image at updated location, cf Eq. (26).

5. Compute the proposed generalized prediction of the displacement resolution, $c f$ Eq.(50).

\section{Conclusion}

The revisited version of the resolution prediction associated with DIC DoF, which is introduced in this paper, gives a better estimation than the usual formula that exists in the literature. The effect due to the interpolation of the back-deformed image $\left(g_{\mathrm{BD}}\right)$ that occurs for sub-pixel displacements is indeed correctly taken into account whereas it is usually omitted. Moreover, this prediction of the resolution is here generalized for DIC outputs directly on displacement maps which are defined pixelwise. It can be applied to all cases of DIC since it is not reduced to FE-based DIC, as in $[18,12]$. Finally, a careful design of a noiseless DIC tangent operator permits to improve the resolution of DIC output and also to decrease the bias of the prediction. Nevertheless, the proposed formula slightly overestimates the resolution, because of the simplified assumption still needed in our calculations. An experimental validation of the introduced prediction is proposed in [4]. It is focused on the particular pre-processing tools that need to be applied to the images, in order to make experimental data to satisfy the hypothesis needed by the predictive formula. 


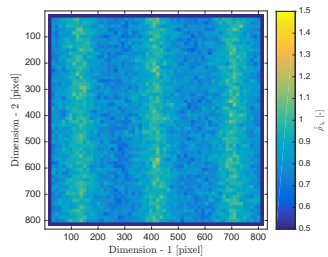

(a) Ratio $\hat{\hat{\rho}}_{\lambda}$ (map)

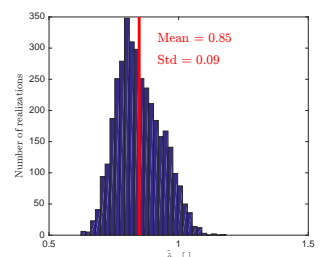

(e) Ratio $\hat{\hat{\rho}}_{\lambda}$ (histogram)

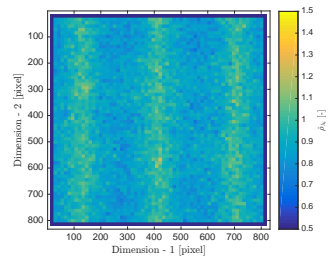

(b) Ratio $\hat{\rho}_{\lambda}$ (map)

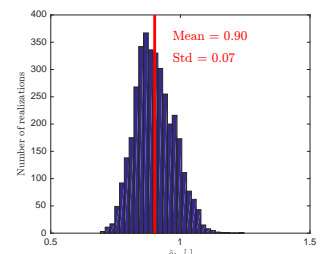

(f) Ratio $\hat{\rho}_{\lambda}$ (histogram)

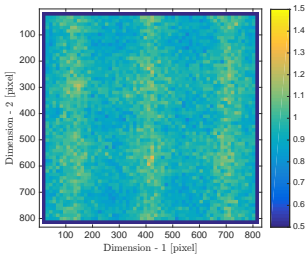

(c) Ratio $\bar{\rho}_{\lambda}$ (map)

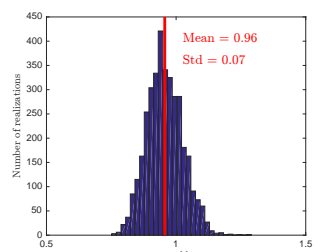

(g) Ratio $\bar{\rho}_{\lambda}$ (histogram)

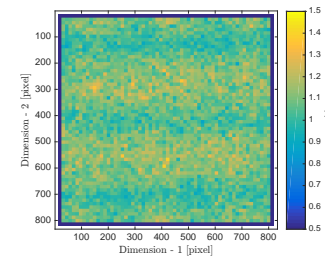

(d) Ratio $\rho_{\lambda}$ (map)

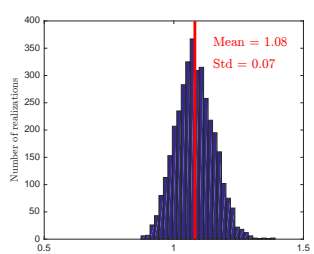

(h) Ratio $\rho_{\lambda}$ (histogram)

Figure 9: Observation and predictions of the vertical displacement standard deviation

\section{Acknowledgments}

The research group "GDR - ISIS" (CNRS) is gratefully acknowledged for its financial support (project "TIMEX").

\section{References}

[1] B. K. Bay, T. S. Smith, D. P. Fyhrie, and M. Saad. Digital volume correlation: Threedimensional strain mapping using x-ray tomography. Experimental Mechanics, 39(3):217226, 1999.

[2] S. Bergonnier, F. Hild, and S. Roux. Digital image correlation used for mechanical tests on crimped glass wool samples. Journal of Strain Analysis for Engineering Design, 40(2):185197, 2005.

[3] G. Besnard, F. Hild, and S. Roux. Finite-element displacement fields analysis from digital images: application to Portevin-Le Châtelier bands. Experimental Mechanics, 46(6):789803, 2006.

[4] B. Blaysat, M. Grédiac, and F. Sur. On the propagation of camera sensor noise to displacement maps obtained by DIC - an experimental study. Experimental Mechanics, 2016. To be published.

[5] B. Blaysat, J. P. M. Hoefnagels, G. Lubineau, M. Alfano, and M. G. D. Geers. Interface debonding characterization by image correlation integrated with double cantilever beam kinematics. International Journal of Solids and Structures, 55:79-91, 2015. 
[6] T. C. Chu, W. F. Ranson, and M. A. Sutton. Applications of Digital-Image-Correlation techniques to experimental mechanics. Experimental Mechanics, 25(3):232-244, 1985.

[7] G. L. G. Gonzáles and J. L. F. Freire. Evaluation of accuracy and spatial resolution in high strain gradient fields. In 1st International Digital Image Correlation Society Conference and Workshop, Columbia, S.C., U.S.A., 2015.

[8] R. Gras, H. Leclerc, F. Hild, S. Roux, and J. Schneider. Identification of a set of macroscopic elastic parameters in a 3D woven composite: Uncertainty analysis and regularization. International Journal of Solids and Structures, 55:2-16, 2015.

[9] M. Grédiac and F. Hild. Full-field measurements and identification in solid mechanics. Wiley, 2013.

[10] M. Grédiac and F. Sur. Effect of sensor noise on the resolution and spatial resolution of displacement and strain maps estimated with the grid method. Strain, 50(1):1-27, 2014.

[11] A. Gut. An Intermediate Course in Probability. Springer, 2009.

[12] F. Hild and S. Roux. Comparison of local and global approaches to digital image correlation. Experimental Mechanics, 52:1503-1519, 2012.

[13] JCGM Member Organizations. International vocabulary of metrology - Basic and general concepts and associated terms (VIM), volume 200. BIPM, 2012.

[14] X. D. Ke, H. W. Schreier, M. A. Sutton, and Y. Q. Wang. On error assessment in stereobased deformation measurements. Experimental Mechanics, 51(4):423-441, 2011.

[15] J. Neggers, B. Blaysat, J. P. M. Hoefnagels, and M. G. D. Geers. On image gradients in digital image correlation. International Journal for Numerical Methods in Engineering, 105(4):243-260, 2016.

[16] B. Pan, B. Wang, G. Lubineau, and A. Moussawi. Comparison of subset-based local and finite element-based global digital image correlation. Experimental Mechanics, 55(5):887$901,2015$.

[17] J. Réthoré. A fully integrated noise robust strategy for the identification of constitutive laws from digital images. International Journal for Numerical Methods in Engineering, 84(6):631-660, 2010.

[18] J. Réthoré, G. Besnard, G. Vivier, F. Hild, and S. Roux. Experimental investigation of localized phenomena using digital image correlation. Philosophical Magazine, 88(28-29):3339$3355,2008$.

[19] J. Réthoré, F. Hild, and S. Roux. Extended digital image correlation with crack shape optimization. International Journal for Numerical Methods in Engineering, 73(2):248-272, 2008.

[20] S. Roux and F. Hild. Stress intensity factor measurements from digital image correlation: post-processing and integrated approaches. International Journal of Fracture, 140(1):141$157,2006$.

[21] H. Schreier, J.-J. Orteu, and M. A. Sutton. Image correlation for shape, motion and deformation measurements: Basic Concepts, Theory and Applications. Springer, 2009. 
[22] E. P. Simoncelli. Handbook of Computer Vision and Applications, chapter Bayesian MultiScale Differential Optical Flow. Springer, 1998.

[23] F. Sur and M. Grédiac. Sensor noise modeling by stacking pseudo-periodic grid images affected by vibrations. IEEE Signal Processing Letters, 21(4):432-436, 2014.

[24] F. Sur, N. Noury, and M.-O. Berger. Computing the uncertainty of the 8 point algorithm for fundamental matrix estimation. In Proceedings of the British Machine Vision Conference (BMVC), volume 2, pages 965-974, Leeds (UK), September 2008.

[25] M. A. Sutton, S. R. McNeill, J. Jang, and M. Babai. Effects of subpixel image restoration on digital correlation error estimates. Optical Engineering, 27(10):271070, 1988.

[26] M. A. Sutton, C. Mingqi, W. H. Peters, Y. J. Chao, and S. R. McNeill. Application of an optimized digital correlation method to planar deformation analysis. Image and Vision Computing, 4(3):143 - 150, 1986.

[27] W. Tong. Formulation of Lucas-Kanade digital image correlation algorithms for non-contact deformation measurements: A review. Strain, 49(4):313-334, 2013.

[28] W. Tong. Reduction of noise-induced bias in displacement estimation by linear off-pixel digital image correlation. Strain, 49(2):158-166, 2013.

[29] G. Vendroux and W. G. Knauss. Submicron deformation field measurements: Part 2. improved digital image correlation. Experimental Mechanics, 38(2):86-92, 1998.

[30] Y. Q. Wang, M. A. Sutton, H. A. Bruck, and H. W. Schreier. Quantitative error assessment in pattern matching: Effects of intensity pattern noise, interpolation, strain and image contrast on motion measurements. Strain, 45(2):160-178, 2009.

[31] Y. Q. Wang, M. A. Sutton, X. D. Ke, H. W. Schreier, P. L. Reu, and T. J. Miller. On error assessment in stereo-based deformation measurements. Experimental Mechanics, 51(4):405$422,2011$.

[32] Z. Y. Wang, H. Q. Li, J. W. Tong, and J. T. Ruan. Statistical analysis of the effect of intensity pattern noise on the displacement measurement precision of digital image correlation using self-correlated images. Experimental Mechanics, 47(5):701-707, 2007.

[33] L. Wittevrongel, P. Lava, S. V. Lomov, and D. Debruyne. A self adaptive global digital image correlation algorithm. Experimental Mechanics, 55(2):361-378, 2015.

[34] J. Xu, A. Moussawi, R. Gras, and G. Lubineau. Using image gradients to improve robustness of digital image correlation to non-uniform illumination: effects of weighting and normalization choices. Experimental Mechanics, 55(5):963-979, 2015. 